\title{
Article \\ Thermodynamic Modeling of the Uranium-Tellurium System: Estimation of the Uncertainties by a Bayesian Approach
}

\author{
Christine Guéneau ${ }^{1, *}$, Eva Lawrence ${ }^{1,2}$, Thierry Klein ${ }^{2,3}$ and Fabrice Gamboa ${ }^{2}$ (D) \\ 1 Université Paris-Saclay, CEA, Service de la Corrosion et du Comportement des Matériaux dans leur \\ Environnement, 91191 Gif-sur-Yvette, France; evalawrence.pro@gmail.com \\ 2 Institut de Mathématiques de Toulouse, 31400 Toulouse, France; thierry.klein@math.univ-toulouse.fr (T.K.); \\ fabrice.gamboa@math.univ-toulouse.fr (F.G.) \\ 3 Ecole Nationale d'Aviation Civile, 31400 Toulouse, France \\ * Correspondence: christine.gueneau@cea.fr
}

check for

updates

Citation: Guéneau, C.; Lawrence, E.; Klein, T.; Gamboa, F.

Thermodynamic Modeling of the Uranium-Tellurium System:

Estimation of the Uncertainties by a Bayesian Approach. Thermo 2022, 2 15-38. https://doi.org/10.3390/ thermo2010003

Academic Editor: Johan Jacquemin

Received: 6 January 2022

Accepted: 5 February 2022

Published: 16 February 2022

Publisher's Note: MDPI stays neutral with regard to jurisdictional claims in published maps and institutional affiliations.

Copyright: (C) 2022 by the authors. Licensee MDPI, Basel, Switzerland. This article is an open access article distributed under the terms and conditions of the Creative Commons Attribution (CC BY) license (https:// creativecommons.org/licenses/by/ $4.0 /)$.

\begin{abstract}
Under irradiation, the formation of fission products in the $(\mathrm{U}, \mathrm{Pu}) \mathrm{O}_{2}$ fuel with time has a substantial effect on its chemistry. In particular, migration of the most volatile fission products (Cs, Te, I, Mo) from the center to the periphery of the fuel pellet is induced by the large radial thermal gradient. To predict the thermodynamic properties of the irradiated fuel, thermodynamic modeling of the complex multi-component (Cs-I-Te-Mo)-(U-Pu)-O system is performed using the CALPHAD method. In this work, the thermodynamic assessment of the U-Te sub-system is performed. The literature review reveals a lack of experimental data as well as scattering and inconsistency of some of the data. In particular, no thermodynamic data exist on the liquid. From this review, input thermodynamic and phase diagram data are carefully selected. The Gibbs energy functions are then adjusted by fitting these data. An overall good agreement is obtained with all the selected data except for the enthalpy of formation for UTe which is underestimated by $13 \%$ by our model. This could be due to an inconsistency between the enthalpy of formation and vapor pressure data. In a second step, the uncertainties on the thermodynamic parameters and their propagation on the calculated thermodynamic and phase diagram data are estimated using a Bayesian approach. The analysis shows that there are too many parameters (22) for too few data points (120 points). The uncertainties are thus large on some of the calculated data. Moreover the inconsistency of some of the data and the lack of thermodynamic data for the liquid makes the model uncertain. New experimental data such as heat capacity, enthalpy of formation for the compounds, and chemical potentials or activities for the liquid phase would improve the reliability of our model. Measurements of phase diagram data in the $\mathrm{U}-\mathrm{UTe}_{2}$ region are also required. However this work provides the first detailed uncertainty analysis of the U-Te CALPHAD model. Moreover our approach, contrary to other Bayesian methods, provides an analytical posterior probability distribution and analytical credible intervals for the calculated thermodynamic quantities. It also speeds up the simulation of the uncertainty estimations on the phase diagram.
\end{abstract}

Keywords: uranium; tellurium; thermodynamic; modeling; CALPHAD; uncertainties; Bayesian

\section{Introduction}

In sodium-cooled fast reactors (SFR), the fuel pins are made of uranium and plutonium dioxide $(\mathrm{U}, \mathrm{Pu}) \mathrm{O}_{2}$ pellets stacked in steel cladding. These fuel pellets undergo a large radial thermal gradient with a temperature of about $2200 \mathrm{~K}$ in the center and $850 \mathrm{~K}$ in the rim. Numerous fission products (FPs) are produced in the fuel through the fission nuclear reactions. Among these fission products, the most volatile ones such as iodine, cesium, tellurium, and molybdenum migrate from the center to the periphery of the fuel pellet and form a layer enriched in these fission products, the so-called "Joint-Oxyde-Gaine" or JOG [1,2]. With time, these fission products can attack the steel cladding to form the so-called "Reaction-Oxyde-Gaine" or ROG, which is a limiting factor for the cladding 
lifetime. To supply the models predicting the JOG and ROG layer thickness, the TAF-ID (Thermodynamics of Advanced Fuels-International Database [3]) thermodynamic database is being developed on the (U-Pu-O)-(I-Cs-Te-Mo)-(Fe-Cr-Ni) system using the CALPHAD method $[2,3]$.

In the CALPHAD method [4], the Gibbs energies of all the phases (solids, liquid, gas) in the binary and ternary sub-systems are described as a function of temperature, composition, and pressure (only for the gas phase). By extrapolation from the sub-systems, the thermodynamic equilibrium in a multi-component system can then be calculated by minimizing the total Gibbs energy of the system. This powerful method has been extensively used since the ' 80 s to calculate phase equilibria and thermodynamic properties of multi-component systems. In these models, the thermodynamic parameters are assessed by fitting the available phase diagram and thermodynamic data. Nevertheless, the propagation of these fitting data uncertainties onto the thermodynamic parameters featured in the Gibbs energy functions in the CALPHAD models are rarely estimated. A few works have been published on this topic, see [5-7] for the most recent analyses. Moreover the propagation of these uncertainties on the calculated phase diagram and thermodynamic data are almost never determined. However it is crucial to estimate these uncertainties, in particular when these models are used in multi-physics codes like for instance the Fuel Performance Codes (ALCYONE and GERMINAL) which is used to simulate the thermomechanical behavior of a fuel pin [1,2]. The influence of these uncertainties on the physical properties and the behavior of the fuel (margin to the melting, thermal conductivity, ...) has to be studied.

In the present work, the thermodynamic modeling of the U-Te binary sub-system is first performed using the PARROT module in the Thermo-Calc software [8]. In a second step, a Bayesian approach is applied to estimate the uncertainties on the thermodynamic parameters and their propagation on the calculated phase diagram and thermodynamic data.

The state of the art on the crystal structure data of the $\mathrm{U}_{x} \mathrm{Te}_{y}$ compounds, the phase diagram, and thermodynamic data is first presented in Section 2. The models to describe the Gibbs energy of the phases are described in Section 3. From these selected data and Gibbs energy models, a least-square method is applied to assess the Gibbs energy functions of the phases. In Section 4, the results of the fitting procedure are presented and the uncertainties on the assessed thermodynamic parameters are estimated using a Bayesian approach. Their propagation on the calculated thermodynamic and phase diagram data is then described. Finally, all the results are discussed.

\section{State of the Art on the U-Te System and Selection of the Data for the Assessment}

In this section, the crystal structure and thermodynamic data on the $\mathrm{U}_{x} \mathrm{Te}_{y}$ compounds and the phase diagram information are reviewed. From this critical review, the input data for the optimization procedure are selected.

\subsection{Crystal Structure Data}

Numerous compounds exist in the U-Te system: $\mathrm{U}_{10} \mathrm{Te}, \mathrm{UTe}, \mathrm{U}_{3} \mathrm{Te}_{4}, \mathrm{U}_{2} \mathrm{Te}_{3}, \mathrm{U}_{3} \mathrm{Te}_{5}$, $\mathrm{U}_{7} \mathrm{Te}_{12}, \beta-\mathrm{UTe}_{2}, \alpha-\mathrm{UTe}_{2}, \mathrm{U}_{2} \mathrm{Te}_{5}, \mathrm{UTe}_{3}, \mathrm{U}_{2} \mathrm{Te}_{7}, \mathrm{UTe}_{5}$. In 1993, Okamoto [9] reviewed the crystal structure data on the compounds. The data are reported in Table 1 based on the most recent works of Hermannsdörfer et al. [10] for UTe, Tougait et al. [11] for $\mathrm{U}_{2} \mathrm{Te}_{3}$ and $\mathrm{U}_{3} \mathrm{Te}_{5}$ and Stöwe [12] for $\alpha-\mathrm{UTe}_{2}$ and $\mathrm{UTe}_{3}$.

In the review by Okamoto [9], $\mathrm{U}_{7} \mathrm{Te}_{12}$ is reported instead of $\mathrm{U}_{3} \mathrm{Te}_{5}$ in the phase diagram. The author argued that the structure of $\mathrm{U}_{3} \mathrm{Te}_{5}$ was not well defined. Later, Tougait et al. [11] investigated the crystal structure of both $\mathrm{U}_{3} \mathrm{Te}_{5}$ and $\mathrm{U}_{2} \mathrm{Te}_{3}$ by neutron diffraction. Thus based on these updated data, the compound $\mathrm{U}_{3} \mathrm{Te}_{5}$ is finally considered instead of $\mathrm{U}_{7} \mathrm{Te}_{12}$. Nevertheless, it is not clear whether this compound is stoichiometric or exhibits a small composition range. Due to the lack of reliable data, $\mathrm{U}_{3} \mathrm{Te}_{5}$ will be considered as a stoichiometric compound in the present work. 
The high temperature $\beta-\mathrm{UTe} e_{2}$ form was reported in the phase diagram of Slovyanskikh et al. [13] whose experimental work clearly showed an allotropic transition for this compound. However, the crystal structure of the $\beta$ form is not known.

The possible existence of $U_{2} \mathrm{Te}_{7}$ was reported in the phase diagram of Okamoto [9]. However, due to the lack of data, it is not considered in the present work.

The compounds highlighted in bold in Table 1 are considered in our model. Due to the lack of reliable data on compounds composition range, only stoichiometric compounds are considered in the present work.

Table 1. Crystal structure data on the phases of the U-Te system. The compounds in bold are considered in the present work. The compounds in italic gray ${ }^{\star}$ are not taken into account.

\begin{tabular}{|c|c|c|c|}
\hline Compounds & Composition At. \% Te & Space Group & Reference \\
\hline$\gamma-U$ & 0 & $\operatorname{Im} \overline{3} \mathrm{~m}$ & Okamoto (1993) [9] \\
\hline$\beta-U$ & 0 & $\mathbf{P 4}_{2} / \mathbf{m m m}$ & Okamoto (1993) [9] \\
\hline$\alpha-U$ & 0 & Clcl & Okamoto (1993) [9] \\
\hline$U_{10} T e^{\star}$ & 9 & $F m-3 m$ & Okamoto (1993) [9] \\
\hline UTe & 50 & Fm-3m & Hermannsdörfer et al. (2006) [10] \\
\hline $\mathrm{U}_{3} \mathrm{Te}_{4}$ & 57.1 & I-43d & Solvyanskikh et al. (1977) [14] \\
\hline $\mathrm{U}_{2} \mathrm{Te}_{3}$ & 60 & Pnma & Tougait et al. (2001) [11] \\
\hline $\mathrm{U}_{3} \mathrm{Te}_{5}$ & 62.5 & Pnma & Tougait et al. (2001) [11] \\
\hline$U_{7} \mathrm{Te}_{12}{ }^{*}$ & 63.2 & $P-6$ & Tougait et al. (1998) [15] \\
\hline$\beta$-UTe 2 & 66.7 & Unknown & Okamoto (1993) [9] \\
\hline$\alpha-\mathrm{UTe}_{2}$ & 2 & 2 & Stöwe (1996) [12] \\
\hline $\mathrm{U}_{2} \mathrm{Te}_{5}$ & 71.4 & $\mathrm{C} 12 \mathrm{~m} 1$ & Tougait et al. (1997) [16] \\
\hline $\mathrm{UTe}_{3}$ & 75 & $\mathrm{P} 12_{1} / \mathrm{m} 1$ & Stöwe (1996) [12] \\
\hline$U_{2} \mathrm{Te}_{7}{ }^{\star}$ & 77.8 & Unknown & Okamoto (1993) [9] \\
\hline $\mathrm{UTe}_{5}$ & 83.3 & Pnma & Boehme et al. (1992) [17] \\
\hline $\mathrm{Te}$ & 100 & $\mathrm{P}_{1} 21$ & Okamoto (1993) [9] \\
\hline
\end{tabular}

\subsection{Phase Diagram Data}

The phase diagram data coming from the literature are summarized in Table 2 and reviewed in this section.

Slovyanskikh et al. [13] investigated the UTe-Te region using differential thermal analysis (DTA) and X-ray diffraction. From the DTA measurements, a eutectic reaction between $\mathrm{UTe}_{5}$ and $\mathrm{Te}$ and the peritectic decompositions of $\mathrm{UTe}_{5}, \mathrm{UTe}_{3}, \mathrm{U}_{3} \mathrm{Te}_{7}, \mathrm{UTe}_{2}$, and $\mathrm{U}_{3} \mathrm{Te}_{5}$ were highlighted. A polymorphic transition for $\mathrm{UTe}_{2}$ was also found to occur at $1125^{\circ} \mathrm{C}$. The compounds $\mathrm{U}_{2} \mathrm{Te}_{3}, \mathrm{UTe}_{2}, \mathrm{U}_{3} \mathrm{Te}_{5}, \mathrm{U}_{3} \mathrm{Te}_{7}, \mathrm{UTe}_{3}$, and $\mathrm{UTe}_{5}$ were identified using X-ray diffraction. A small region of homogeneity was assumed for $\mathrm{UTe}_{2}$ and $\mathrm{U}_{3} \mathrm{Te}_{5}$. Finally, liquidus temperatures were carefully measured from $\mathrm{UTe}_{2}$ to $\mathrm{Te}$.

The $\mathrm{U}$ rich region from 0 to 65 at. \% Te was later investigated using the same methods by Ellert et al. (1975) [18]. The melting point of uranium monotelluride was measured at $1730^{\circ} \mathrm{C}$. The authors reported that the temperature of the eutectic between $\mathrm{U}$ and $\mathrm{UTe}$ measured at $1180^{\circ} \mathrm{C}$ was probably overestimated due to a partial dissolution of the crucible material. Liquidus temperatures were also determined. In the UTe-Te region, the authors mention that the results obtained in the range 50-57.2 at. \% Te are uncertain. $\mathrm{The}_{3} \mathrm{U}_{3}$ compound was assumed to decompose by a peritectic reaction at $1540{ }^{\circ} \mathrm{C}$. The range 57.2-60 at. \% Te was found to be a single phase region (with $\mathrm{Th}_{3} \mathrm{P}_{4}$ crystal structure) with $\mathrm{U}_{3} \mathrm{Te}_{4}$ and $\mathrm{U}_{2} \mathrm{Te}_{3}$ as boundary compositions. A peritectic decomposition temperature of $1500{ }^{\circ} \mathrm{C}$ was assumed for $\mathrm{U}_{2} \mathrm{Te}_{3}$. The peritectic decomposition of $\mathrm{U}_{3} \mathrm{Te}_{5}$ was measured at $1300{ }^{\circ} \mathrm{C}$ instead of $1260{ }^{\circ} \mathrm{C}$ by Slovyanskikh et al. [13]. The authors in [13] suggest the existence of a small composition range for $\mathrm{U}_{7} \mathrm{Te}_{12}$ between $63.2-63.8$ at. \% Te given their results with X-ray diffraction. 
In 1985, Czechowicz [19] proposed a phase diagram based on the data of Slovyanskikh et al. [13].

A phase diagram of the U-Te system was later proposed in the review by Okamoto in 1993 [9], mainly based on the experimental data of Ellert et al. (1975) [18]. Only the compounds $U_{7} \mathrm{Te}_{12}$ and $\mathrm{U}_{2} \mathrm{Te}_{5}$ were considered instead of $\mathrm{U}_{3} \mathrm{Te}_{5}$ and $\mathrm{U}_{3} \mathrm{Te}_{7}$. A composition range was also represented for the $\mathrm{UTe}_{2}$ compound.

Recently, in 2017, Wolf [20] performed a thermodynamic assessment of the U-Te system using the Calphad method. The experimental data are not reported on the calculated phase diagram in [20]. However the calculated liquidus curve and the experimental data are not in good agreement.

Table 2. Phase diagram data on the U-Te system.

\begin{tabular}{cccc}
\hline Composition Range At. \% Te & Method & Reference & Comments \\
\hline $58-100$ & DTA, XRD & Slovyanskikh et al. (1968) [13] & Selected \\
$0-65$ & DTA, XRD & Ellert et al. (1975) [18] & Selected \\
$0-100$ & Review & Czechowitz (1985) [19] & Not selected \\
$0-100$ & Review & Okamoto (1993) [9] & Not selected \\
100 & Calphad model & Wolf (2017) [20] & Not selected \\
\hline
\end{tabular}

In conclusion, the phase diagram is well established in the region from $\mathrm{UTe}_{2}$ to pure Te based on Slovyanskikh et al. [13]. However, the decomposition temperatures of the compounds and the liquidus data remain uncertain in the $\mathrm{UTe}-\mathrm{U}_{3} \mathrm{Te}_{5}$ composition range. Moreover the liquidus in the U-UTe part is still not well known. For the optimization procedure, we selected only the experimental data measured by Slovyanskikh et al. [13] and Ellert et al. [18]. The compounds (in bold in Table 1) were selected and considered as stoichiometric in the present work.

\subsection{Thermodynamic Data}

All the data are reported in Table 3. No data exist on the liquid phase and very few measurements were performed on the solid compounds.

Westrum [21] estimated the standard entropies of $\mathrm{UTe}, \mathrm{U}_{3} \mathrm{Te}_{4}, \mathrm{U}_{2} \mathrm{Te}_{3}, \mathrm{UTe}_{2}, \mathrm{U}_{2} \mathrm{Te}_{5}$, and $\mathrm{UTe}_{3}$ compounds.

There are not any heat capacity measurements. Only estimations are provided by Czechowicz [19] and Wolf [20]. The estimation values from Czechowicz [19] are significantly higher than those from Wolf [20] at high temperatures. Due to the lack of experimental data, the $C_{p}(T)$ data of Wolf [20] based on the Kopp-Neumann rule are considered in the present work for all compounds.

All the enthalpy of formation data are reported in Figure 1. The enthalpy of formation for $U T e$ and $\mathrm{U}_{3} \mathrm{Te}_{4}$ was measured by direct-reaction calorimetry using a Calvettype high-temperature liquid metal (tin) solution calorimeter. The values are equal to: $-182.4 \pm 11.3 \mathrm{~kJ} / \mathrm{mol}$ for UTe and $-228.0 \pm 1.3 \mathrm{~kJ} / \mathrm{mol}$ for $\mathrm{UTe}_{1.33}$. The enthalpy of formation for $\mathrm{UTe}_{2}$ was also estimated to be $-318 \mathrm{~kJ} / \mathrm{mol}$ by the authors assuming a linear relation to the $\mathrm{Te} / \mathrm{U}$ ratio.

Czechowicz [22] reported uncertainties in the work of Baskin et al. [23] due to the uncertain percentage of each phase in the samples for calorimetric measurements. Later Mills [24] reviewed the data and assigned them larger uncertainties: $-182.4 \pm 20.9 \mathrm{~kJ} / \mathrm{mol}$ for $\mathrm{UTe}$ and $-684.1 \pm 83.7 \mathrm{~kJ} / \mathrm{mol}$ for $\mathrm{U}_{3} \mathrm{Te}_{4}$. These solely experimental data of Baskin et al. [23] for $\mathrm{UTe}, \mathrm{U}_{3} \mathrm{Te}_{4}$ are selected in this work. 


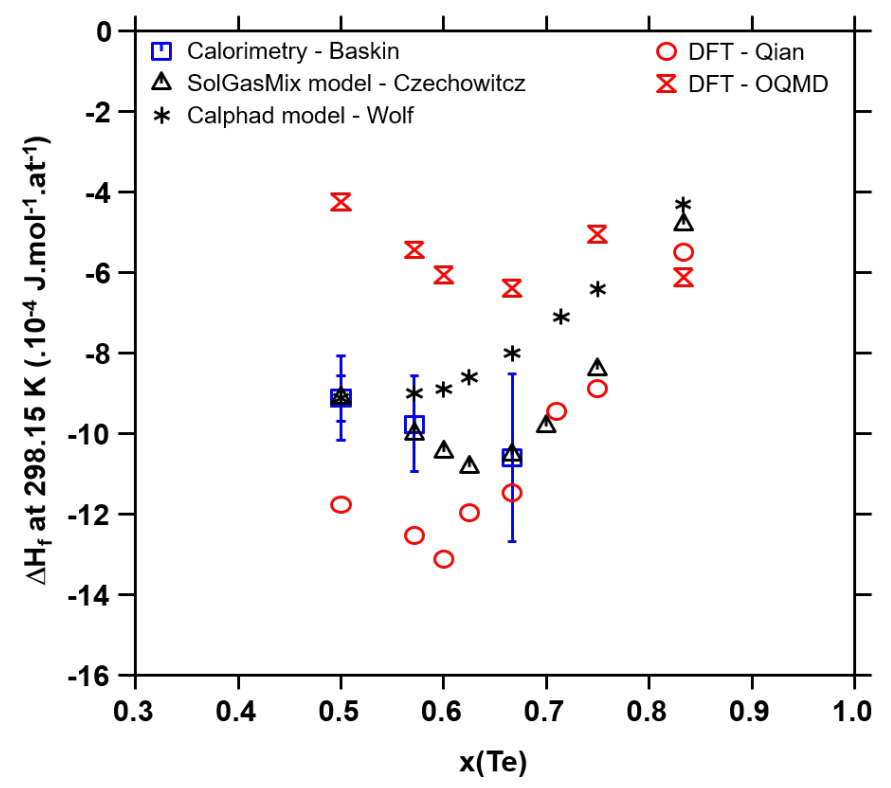

Figure 1. Enthalpy of formation data of the $\mathrm{U}_{x} \mathrm{Te}_{y}$ compounds coming from the literature. The uncertainty bars on the experimental data measured by calorimetry (in blue) are reported.

There are no other enthalpy of formation measurements. However in 1985, Czechowicz [19] published estimated thermodynamic data $\left(\Delta H_{f, 298.15 K}^{0}, S_{298.15 K}^{0}, C_{p}(T), \Delta H_{\text {fusion }}\right)$ on $\mathrm{UTe}, \mathrm{U}_{3} \mathrm{Te}_{4}, \mathrm{U}_{2} \mathrm{Te}_{3}, \mathrm{U}_{3} \mathrm{Te}_{5}, \mathrm{UTe}_{2}, \mathrm{U}_{3} \mathrm{Te}_{7}, \mathrm{UTe}_{3}$, and $\mathrm{UTe}_{5}$. The authors only mentioned that the thermodynamic data were obtained from the literature or estimated without any further explanation. Recently Wolf [20] derived a Calphad model on U-Te. The authors also reported thermodynamic data $\left(\Delta H_{f, 298.15 K}^{0}, S_{298.15 K}^{0}, C_{p}(T)\right)$ for the compounds. For all the compounds except UTe and $\mathrm{U}_{3} \mathrm{Te}_{4}$, the data of Czechowicz [19] were selected. The data of Wolf [20] were discarded due to the disagreement with the data of Baskin et al. [23] for $\mathrm{U}_{3} \mathrm{Te}_{4}$.

More recently, density functional theory (DFT) calculations were performed on U-Te compounds by Qian et al. [25]. Additionally, other DFT data are available in the OQMD database [26]. Very large discrepancies are found between both DFT studies. Moreover none of these studies are in agreement with the experimental data of Baskin et al. [23] for $\mathrm{UTe}, \mathrm{U}_{3} \mathrm{Te}_{4}$, and $\mathrm{UTe}_{2}$. Therefore the DFT data were not used.

Measurements of $\mathrm{Te}_{2}$ equilibrium vapor pressure were performed in two phase regions: $\mathrm{UTe}_{3}-\mathrm{U}_{2} \mathrm{Te}_{5}$ by Slovyanskikh et al. [27] using a manometer, $\mathrm{UTe}_{2} / \mathrm{U}_{3} \mathrm{Te}_{5}$ by Sevast'yanov et al. [28] and in $\mathrm{U}_{3} \mathrm{Te}_{4} / \mathrm{UTe}$ by Czechowicz [22] by high temperature mass spectrometry. Czechowicz [22] provided a detailed description of their experimental conditions and results analysis. The authors reported the inconsistency of their results with the enthalpy of formation data of Baskin et al. [23]. However the large uncertainties in the experimental work of Baskin et al. [23] due to the unknown percentage of the phases in the calorimetric products was mentioned. All the vapor pressure data were used in the present work.

In conclusion, for the optimization procedure, we selected the heat capacity functions derived by Wolf [20], the experimental data of Baskin et al. [23] for the enthalpy of formation of $\mathrm{UTe}$ and $\mathrm{U}_{3} \mathrm{Te}_{4}$ and the estimated data of Czechowicz [19] for the other compounds. The only existing standard entropies estimated by Westrum [21] were also considered. Finally all the $\mathrm{Te}_{2}$ partial pressure data measurements by Slovyanskikh et al. [27], Sevast'yanov et al. [28], and Czechowicz [22] were used. 
Table 3. Thermodynamic data on the phases of the U-Te system. *: the condensed phases were not characterized. The reported phases by Czechowicz 1986 [22] were proposed on the basis of the phase diagram. The method DFT+U refers to the density functional theory + Hubbard model.

\begin{tabular}{|c|c|c|c|c|}
\hline Thermodynamic Data & Phases & Method & Reference & Comments \\
\hline$S_{298.15 K}^{0}$ & $\begin{array}{l}U T e_{2}, U_{3} T_{4}, U_{2} T e_{3} \\
U T e_{2}, U_{2} T e_{5}, U T e_{3}\end{array}$ & Estimation & Westrum 1962 [21] & Selected \\
\hline$\Delta H_{f, 298.15 K}^{0}$ & $U T e, U_{3} \mathrm{Te}_{4}$ & $\begin{array}{l}\text { Direct reaction } \\
\text { calorimetry }\end{array}$ & $\begin{array}{c}\text { Baskin and Smith } \\
1970[23]\end{array}$ & Selected \\
\hline $\begin{array}{c}\Delta H_{f, 298.15 K^{\prime}}^{0} S_{298.15 K}^{0} \\
C_{p}(T), \Delta H_{\text {fusion }}\end{array}$ & $\begin{array}{l}U T e, U_{3} T e_{4}, U_{2} T e_{3}, U_{3} T e_{5} \\
U T e_{2}, U_{2} T e_{5}, U T e_{3}, U T e_{5}\end{array}$ & Estimation Solgasmix & Czechowicz 1985 [19] & $\begin{array}{c}\Delta H_{f, 298.15 K}^{0} \text { selected } \\
\text { except } U T e \text { and } U_{3} T e_{4}\end{array}$ \\
\hline$p_{\mathrm{Te}_{2}}$ & ${ }^{*} U \mathrm{Te}_{3} / U_{2} \mathrm{Te}_{5}$ & $\begin{array}{l}\text { Quartz membrane } \\
\text { null manometer } \\
\text { Chemical transport } \\
\text { reaction }\end{array}$ & $\begin{array}{l}\text { Slovyanskikh et al., } \\
1967 \text { [27] }\end{array}$ & Selected \\
\hline$p_{\mathrm{Te}_{2}}$ & ${ }^{*} U T e_{2} / U_{3} \mathrm{Te}_{5}$ & $\begin{array}{l}\text { Bourdon null } \\
\text { manometer } \\
\text { Chemical transport } \\
\text { reaction }\end{array}$ & $\begin{array}{c}\text { Sevast'yanov et al., } \\
1971[28]\end{array}$ & Selected \\
\hline$p_{\mathrm{Te}_{1}}, p_{\mathrm{Te}_{2}}$ & $U_{3} \mathrm{Te}_{4} / \mathrm{UTe}$ & KEMS & Czechowicz 1986 [22] & Selected \\
\hline $\begin{array}{c}\Delta H_{f, 298.15 K^{\prime}}^{0} S_{298.15 K}^{0} \\
C_{p}(T)\end{array}$ & $\begin{array}{l}U T e, U_{3} T e_{4}, U_{2} T e_{3}, U_{3} T e_{5} \\
U T e_{2}, U_{2} T e_{5}, U T e_{3}, U T e_{5}\end{array}$ & Calphad model & Wolf 2017 [20] & Only $C_{p}(T)$ selected \\
\hline$\Delta H_{f, 0}^{0}$ & $\begin{array}{c}U T e_{1} U_{3} T_{4}, U_{2} T e_{3} \\
U_{3} T e_{5}, U_{7} T e_{12}, U T e_{2} \\
U_{2} T e_{5}, U T e_{3}, U T e_{5}\end{array}$ & $\mathrm{DFT}+\mathrm{U}$ & Qian et al., 2021 [25] & Not selected \\
\hline$\Delta H_{f, 0}^{0}$ & $\begin{array}{l}U T e_{1} U_{3} T e_{4}, U_{2} T e_{3}, U_{3} T e_{5} \\
U T e_{2}, U_{2} T e_{5}, U T e_{3}, U T e_{5}\end{array}$ & DFT & OQMD [26] & Not selected \\
\hline
\end{tabular}

\section{Thermodynamic Modeling with the CALPHAD Method}

The Gibbs energy functions for all the phases of the uranium-tellurium system are described. Then the strategy for the optimization procedure with PARROT is explained.

\subsection{Gibbs Energy Models}

All the Gibbs energy functions are referred to the enthalpy of the pure elements in their stable state at $298.15 \mathrm{~K}$ and $1 \mathrm{~atm}$ (designated as $H_{i}^{S E R}$ ). For the pure elements $\mathrm{U}$ and Te in their different states, the Gibbs energy functions are taken from the SGTE pure database [29].

For all the stoichiometric compounds, the Gibbs energy function is expressed as follows

$$
G_{m}^{\phi}-\sum_{i} b_{i}^{\phi} H_{i}^{S E R}=a_{0}+a_{1} T+a_{2} T \ln (T)+a_{3} T^{2}+a_{4} T^{-1}
$$

where $b_{i}^{\phi}$ is the stoichiometric factor of element $\mathrm{i}$ in the compound $\phi$ and $a_{n}$ are unknown parameters to be estimated. For the $\mathrm{U}_{x} \mathrm{Te}_{y}$ compounds, the $a_{n}$ coefficients (with $n=2,3,4$ ) are fixed using the $C_{p}(T)$ functions provided by Wolf [20] assuming a Kopp-Neumann law.

For the liquid phase, the ionic two-sublattice model [30] is chosen to keep the consistency with the model for this phase in the TAF-ID database [3]. The sublattice model is the following one

$$
\left(U^{+4}\right)_{P}\left(V a^{-Q}, T e, U T e\right)_{Q} .
$$

In this model, uranium cation $\mathrm{U}^{+4}$ is in the first sublattice and charged vacancies $\left(\mathrm{Va}^{-Q}\right)$, neutral Te and UTe species are introduced in the second sublattice. The site numbers $\mathrm{P}$ and $\mathrm{Q}$ are defined as the average charges of the second and first sublattices, respectively 


$$
\begin{aligned}
& P=Q y_{V a^{-}} \\
& Q=4 .
\end{aligned}
$$

The charge electroneutrality of the liquid phase is maintained by varying the charge $-\mathrm{Q}$ of the vacancies which varies with the composition in Te (or $\mathrm{U}$ ). The Gibbs energy of the liquid phase is then expressed by

$$
\begin{aligned}
G_{m}^{l i q}= & y_{V a} G_{\left(U^{+4}\right)(V a)}^{l i q}+y_{T e} G_{T e}^{l i q}+y_{U T e} G_{U T e}^{l i q}+Q R T\left(y_{V a} \ln y_{V a}+y_{T e} \ln y_{T e}+y_{U T e} \ln y_{U T e}\right) \\
& +y_{V a} y_{U T e}\left(L_{\left(U^{+4}\right)(V a, U T e)}^{0}+\left(y_{V a}-y_{U T e}\right) L_{\left(U^{+4}\right)(V a, U T e)}^{1}+\left(y_{V a}-y_{U T e}\right)^{2} L_{\left(U^{+4}\right)(V a, U T e)}^{2}\right) \\
& +y_{U T e} y_{T e}\left(L_{(U T e, T e)}^{0}+\left(y_{U T e}-y_{T e}\right) L_{(U T e, T e)}^{1}+\left(y_{U T e}-y_{T e}\right)^{2} L_{(U T e, T e)}^{2}\right) .
\end{aligned}
$$

The interaction terms have a linear dependence in temperature defined by

$$
L_{(U T e, T e)}^{i}=b^{i}+c^{i} T
$$

There are three end-members in the model: $\left(\mathrm{U}^{+4}\right)_{1}\left(\mathrm{Va}^{-1}\right)_{4}$ corresponding to pure uranium liquid, pure tellurium liquid, and UTe liquid. The introduction of the UTe species is necessary to reproduce the shape of the phase diagram with a high melting point for UTe and a complex liquidus curve in the UTe-Te part. Moreover, a short-range ordering in the liquid is often observed in the Te-based systems which also justifies the introduction of UTe species even if there are no experimental evidence of its existence.

The interaction terms $L_{\left(U^{+4}\right)(V a, U T e)}^{i}$ and $L_{(U T e, T e)}^{i}$ allow the description of U-UTe and $\mathrm{UTe}-\mathrm{Te}$ parts of the phase diagram, respectively.

Finally, the gas phase is described by an ideal mixing of gaseous species $\left(\mathrm{Te}, \mathrm{Te}_{2}, \mathrm{Te}_{3}\right.$, $\left.\mathrm{Te}_{4}, \mathrm{Te}_{5}, \mathrm{Te}_{6}, \mathrm{Te}_{7}, \mathrm{UTe}, \mathrm{U}\right)$ whose Gibbs energy functions ${ }^{\circ} \mathrm{G}_{i}^{\text {gas }}$ come from [31] for all species except for UTe [32]. The Gibbs energy is expressed as

$$
G^{g a s}=\sum_{i} y_{i}\left[{ }^{o} G_{i}^{g a s}-\sum_{j} b_{i j} H_{j}^{S E R}+R T \ln y_{i}\right]+R T \ln \left(\frac{p}{p_{0}}\right)
$$

where $y_{i}$ are the constituent fractions. Their sum is thus unity. $b_{i j}$ is the number of atoms $j$ in the species $i$. The standard pressure $p_{0}$ is set to $10^{5} \mathrm{~Pa}$. The partial pressure of species $i$ $p_{i}$ is related to the constituent fraction by

$$
p_{i}=y_{i} p
$$

where $p$ is the total pressure.

\subsection{Optimization Procedure}

As previously mentioned, the $C_{p}(T)$ functions provided by Wolf [20] were selected which allowed the $a_{2}, a_{3}$, and $a_{4}$ coefficients to be fixed for all the compounds. As there are no experimental data on the melting enthalpy of UTe, the value from the Solgasmix database published by Czechowicz (1985) [19] was taken.

A first set of parameters $\left(a_{0}, a_{1}\right)$ for UTe was assessed as well as the entropy of melting of the compound using the following data: $\Delta H_{f, 298.15 K}^{0}, S_{298.15 K}^{0}, \Delta H_{f u s i o n}$, and $T_{\text {melting }}$. The other compounds were progressively introduced and interaction parameters in the liquid were assessed to have a starting set of variables. All the compounds were forced to be stable at $298.15 \mathrm{~K}$ by setting their driving forces to be equal to 0 . The optimization was then performed with PARROT to reproduce both the thermodynamic and phase diagram data. The weights on the different experimental data had to be adjusted to get a final good agreement with all the data within the error bars provided by the authors. 


\section{Use of Conjugate Prior Distribution for CALPHAD Modeling}

In this section, the uncertainty quantification on the parameters is studied through a Bayesian approach for the CALPHAD modeling quantities. In the context of the CALPHAD modeling, the integration of the uncertainty study is still at its early stage. However we can quote the works of $[5-7,33,34]$ in which the authors propose different methods to deal with uncertainty management using the Bayesian approach. A wider list of references can be found in ([35] Section III.3.3) as well as a more global description of the methods used in these works.

In the following, we will consider the unknown parameters to be real random quantities and we will denote them with capital letters, that is $A_{0}$ and $A_{1}$ for the random unknown quantities in the Gibbs energy model (1) and $B^{i}$ and $C^{i}$ for the parameters in the interaction term $L^{i}$. In the following, $\mathbb{E}[X]$ denotes the expectation (or mean value) of a real random variable $X$ and $\operatorname{Var}(X)$ its variance, that is respectively the average value of $X$ and the average of the squared distance $(X-\mathbb{E}[X])^{2}$. We also recall that the covariance between two real random variables $X$ and $Y$ is defined by

$$
\operatorname{Cov}(X, Y)=\mathbb{E}[(X-\mathbb{E}[X])(Y-\mathbb{E}[Y])]
$$

When considering a set of several random variables, one can extend the definitions of variance and covariance for this group of variables. This defines the covariance matrix, which is the matrix that features the variance of each random and the pair-wise covariances.

\subsection{Bayesian Inference}

Before getting to the details of the method used in this work, some general description of the Bayesian framework is presented in this section. Notice that we do not provide a comprehensive description here. The interested reader is referred to Robert's book [36] for a more complete description of the Bayesian framework.

Parametric models such as Gibbs energy models (1) are fitted with a sample of data. One can measure the goodness-of-fit with respect to such data via the so-called likelihood function. As we consider a parametric model, the goodness-of-fit value is calculated for given values of the unknown parameters $A_{n}$ and given the fitting data values.

In the Bayesian approach of such parametric models, we consider that there exists a prior knowledge on the parameters of interest $A_{n}$, that is to say that there exists an expert advice about the values that $A_{n}$ can take. The Bayesian inference allows the integration of such anterior knowledge on $A_{n}$. Such knowledge is called the prior probability distribution of the random variables $A_{n}$.

Thanks to Bayes' theorem, these two quantities-the prior probability distribution and the likelihood function - enable to determine the posterior probability distribution. This posterior probability distribution gives the distribution of the parameters values, provided that the experiments results are known. Put in another way, this posterior probability distribution determines what values of the parameters have the most likely outcome when knowing the values of the experiments and their experimental uncertainty.

\subsection{Conjugate Prior Probability Distribution}

The specificity of the approach used here is the use of the so-called conjugate prior probability distribution. Usually, the determination of the posterior probability distribution can be difficult and or numerically expensive. However, there exists some prior probability distribution that works well with some models. It is the case when considering a Gaussian prior probability distribution with a linear model: the Gaussian distribution remains Gaussian when a linear transformation is applied, see for example [37] for more details. This famous result from statistics is well adapted to the case of the CALPHAD modeling as the Gibbs energy model (1) is linear with respect to the parameters $A_{n}$. In this specific case, we can get a Gaussian posterior probability distribution. Notice that, apart from some cases, 
there is no guarantee to obtain an analytical posterior probability distribution. In [35] the author studies the specific use of conjugate prior for the CALPHAD models.

Another interesting result is that a Gaussian distribution is entirely defined by its mean value and its variance. In the case of conjugate prior probability distribution, a closed form expression is known for the posterior mean $v$ and the posterior covariance matrix $\mathbf{S}$. They are given by the following matrix expressions

$$
\mathbf{S}=\left(\mathbf{D}^{t} \mathbf{s}_{\mathbf{Z}}^{-1} \mathbf{D}+\mathbf{s}_{\mathbf{0}}{ }^{-1}\right)^{-1}
$$

and

$$
v=\mathbf{S}\left(\mathbf{D}^{t} \mathbf{s}_{\mathbf{Z}}^{-1} \mathbf{Z}+\mathbf{s}_{\mathbf{0}}{ }^{-1} \boldsymbol{m}_{\mathbf{0}}\right)
$$

where ${ }^{t}$ denotes the matrix transposition operator and where $\mathbf{s}_{\mathbf{Z}}{ }^{-1}$ represents the inverse of the matrix $\mathbf{s}_{\mathbf{Z}}$. In the above expressions, the prior mean and the prior covariance matrix are denoted by $m_{0}$ and $\mathbf{s}_{0}$ respectively, the fitting data are denoted by $Z$ and the experimental uncertainties are denoted by $\mathbf{s}_{\mathbf{Z}}$. Finally, $\mathbf{D}$ is the design matrix, that is the evaluated functions that multiply each parameter. See [35] for more details about the conjugate prior probability distribution approach for the CALPHAD models.

Finally, the adequacy between the number of model parameters and the degree of freedom of the fitting data has been tackled in this work. This study relies on well-known results from linear algebra and is beyond the scope of this paper. However, some details can be found in the Appendix A.

\subsection{Uncertainty on the Calculated Thermodynamic Quantities}

In this section, we explain how to get the posterior probability distribution on calculated thermodynamic data once we obtain the posterior probability distribution of the unknown parameters. We specify how the posterior probability distribution is determined for some specific quantities. Such quantities are the enthalpies of formation for the compounds UTe, $\mathrm{U}_{3} \mathrm{Te}_{4}, \mathrm{U}_{2} \mathrm{Te}_{3}, \mathrm{U}_{3} \mathrm{Te}_{5}, \mathrm{UTe}_{2}, \mathrm{U}_{2} \mathrm{Te}_{5}, \mathrm{UTe}_{3}$ and $\mathrm{UTe}_{5}$; the entropies for $\mathrm{UTe}, \mathrm{U}_{3} \mathrm{Te}_{4}, \mathrm{U}_{2} \mathrm{Te}_{3}$, $\mathrm{UTe}_{2}, \mathrm{U}_{2} \mathrm{Te}_{5}$ and $\mathrm{UTe}_{3}$ compounds and the partial pressures for the two-phases regions $\left(\mathrm{UTe}+\mathrm{U}_{3} \mathrm{Te}_{4}\right),\left(\mathrm{U}_{3} \mathrm{Te}_{5}+\mathrm{UTe} \mathrm{T}_{2}\right)$ and $\left(\mathrm{U}_{2} \mathrm{Te}_{5}+\mathrm{UTe}_{3}\right)$. The results given in the following subsections will be used in Section 5 for the comparisons of the posterior prediction and the posterior prediction error with the experiments.

\subsubsection{Enthalpy of Formation}

Recall the chosen model (1) for the Gibbs energy functions of each compound, it is well known that the enthalpy of formation at temperature $T_{0}=298.15 \mathrm{~K}$ of such compound is given by

$$
\Delta H_{f, T_{0}}^{0}=A_{0}-a_{2} T_{0}-a_{3} T_{0}^{2}+2 a_{4} T_{0}^{-1} .
$$

Recall that the parameters $a_{2}, a_{3}$, and $a_{4}$ are fixed using $C_{p}(T)$ data, one can get the posterior average value and a $95 \%$ credible set for $\Delta H_{f, T_{0}}^{0}$ thanks to well-known results coming from statistics.

The posterior average value of the enthalpy of formation is given by

$$
\mathbb{E}\left[\Delta H_{f, T_{0}}^{0}\right]=v_{0}-a_{2} T_{0}-a_{3} T_{0}^{2}+2 a_{4} T_{0}^{-1}
$$

where $v_{0}$ denotes the posterior mean of random parameter $A_{0}$. The posterior variance of the enthalpy of formation is given by

$$
\operatorname{Var}\left(\Delta H_{f, T_{0}}^{0}\right)=\operatorname{Var}\left(A_{0}\right)=\sigma_{0}^{2}
$$

where $\sigma_{0}^{2}$ denotes the posterior variance of random parameter $A_{0}$.

The two previous quantities can then be used to express a $95 \%$ credible set for the enthalpy of formation. Such intervals allow to display the uncertainty on the calculated 
quantity. The $95 \%$ credible interval is built by using the $2.5 \%$ and the $97.5 \%$ quantiles of the Gaussian standard distribution, namely -1.964 and 1.964, respectively. The following interval is then obtained for $\Delta H_{f, T_{0}}^{0}$

$$
\begin{aligned}
& {\left[\begin{array}{l}
v_{0}-a_{2} T_{0}-a_{3} T_{0}^{2}+2 a_{4} T_{0}^{-1}-1.964 \sigma_{0} \\
v_{0}-a_{2} T_{0}-a_{3} T_{0}^{2}+2 a_{4} T_{0}^{-1}+1.964 \sigma_{0}
\end{array}\right] .}
\end{aligned}
$$

The credible interval (13) is centered and symmetrical with respect to the posterior mean of $\Delta H_{f, T_{0}}^{0}$ (11). This interval is such that it includes the $95 \%$ of $\Delta H_{f, T_{0}}^{0}$ posterior values that have the most likely outcome.

\subsubsection{Entropy}

Recall the chosen model (1) for the Gibbs energy functions of each compound $\phi$, the entropy at temperature $T_{0}=298.15 \mathrm{~K}$ of such compound is given by

$$
S_{T_{0}}^{0}=-A_{1}-a_{2}\left(1+\ln T_{0}\right)-2 a_{3} T_{0}+a_{4} T_{0}^{-2} .
$$

Using the same method as for $\Delta H_{f, T_{0}}^{0}$, one gets the following expressions

$$
\mathbb{E}\left[S_{T_{0}}^{0}\right]=-v_{1}-a_{2}\left(1+\ln T_{0}\right)-2 a_{3} T_{0}+a_{4} T_{0}^{-2}
$$

and

$$
\operatorname{Var}\left(S_{T_{0}}^{0}\right)=\operatorname{Var}\left(A_{1}\right)=\sigma_{1}^{2}
$$

where $v_{1}$ and $\sigma_{1}^{2}$ are respectively the posterior mean and the posterior variance of parameter $A_{1}$. In the same way, one defines the $95 \%$ credible set for the entropy

$$
\begin{aligned}
& {\left[-v_{1}-a_{2}\left(1+\ln T_{0}\right)-2 a_{3} T_{0}+a_{4} T_{0}^{-2}-1.964 \sigma_{1}\right.} \\
& \left.\quad-v_{1}-a_{2}\left(1+\ln T_{0}\right)-2 a_{3} T_{0}+a_{4} T_{0}^{-2}+1.964 \sigma_{1}\right] .
\end{aligned}
$$

4.3.3. Partial Pressures for the Two-Phase Regions

The Gibbs energy function is related to the partial pressure by the relation

$$
\Delta G^{r}=-R T \ln \left(p_{i}\right)
$$

for each value of temperature $T$. The specificity of the partial pressures data is that they are related to several compounds. For example, in the case of the partial pressures for the two-phase region $\left(\mathrm{UTe}+\mathrm{U}_{3} \mathrm{Te}_{4}\right)$, the parameters of $\mathrm{UTe}$ and $\mathrm{U}_{3} \mathrm{Te}_{4}$ are both involved. In this example, we have

$$
\Delta G^{r}=3 G^{U T e}+G^{T e(g)}-G^{U_{3} T e_{4}} .
$$

As in the case of the enthalpy of formation and the entropy, the posterior mean of $\Delta G^{r}$ is simply obtained by replacing the unknown random parameters by their posterior means. This is due to the linearity of the expectation. However, the variance expression is slightly more complicated. Given a temperature $T_{1}$, we denote by $\mathbf{F}$ the following line-vector

$$
\mathbf{F}=\left(\begin{array}{llll}
3 & 3 T_{1} & -1 & -T_{1}
\end{array}\right) .
$$


For this given temperature, the variance of $\Delta G^{r}$ is given by

$$
\operatorname{Var}\left(\Delta G^{r}\right)=\mathbf{F} \mathbf{S}^{\left(U T e+U_{3} T e_{4}\right)} \mathbf{F}^{t}
$$

where the matrix $\mathbf{S}^{\left(U T e+U_{3} T e_{4}\right)}$ is the covariance matrix related to the unknown parameters for phases $\mathrm{UTe}$ and $\mathrm{U}_{3} \mathrm{Te}_{4}$.

As the assessor is usually interested in the uncertainty for an interval of temperatures, the previous expression must be computed for each targeted temperature within the interval range.

\subsection{Uncertatinty on the Calculated Phase Diagram}

Once having at hand the posterior probability distribution of all parameters $A_{n}$, the uncertainty for phase diagram data can be estimated through Monte-Carlo simulations. The method consists in sampling a great number $N$ of the parameters $A_{n}$ values from the posterior probability distribution. For each set of parameters values, the associated phase diagram can be computed. The uncertainty on each phase transition can then be experimentally determined.

Notice that, unlike the uncertainty on the thermodynamic quantities, one can not give an analytical credible interval for the phase transitions. This is due to the fact that the phase transitions are obtained by minimizing the Gibbs energy functions for a given experimental condition. The phase diagram does not depend linearly on the model parameters and therefore, it is not possible to obtain an analytical posterior probability distribution for the phase transitions.

\section{Results and Discussion}

\subsection{CALPHAD Assessment} Table 4

The assessed thermodynamic parameters of the phases using PARROT are reported in

The calculated phase diagram is compared to the experimental data in Figures 2 and 3. In the UTe-Te region (Figure 3), a very good agreement is obtained with the experimental data. The liquidus curve and the invariant reactions are very well reproduced from $\mathrm{UTe}_{2}$ to Te. Only the plateau related to the polymorphic transition of $\mathrm{UTe}_{2}$ was not described in the present assessment as the transition enthalpy of the compound is unknown. The liquidus curve from UTe to $\mathrm{UTe}_{2}$ and the temperature of the peritectic decomposition of $\mathrm{U}_{3} \mathrm{Te}_{4}$ and $\mathrm{U}_{2} \mathrm{Te}_{3}$ remain uncertain due to very few scattered experimental data in this region. In the U-UTe region, the experimental data are scarce so the liquidus curve shape remains quite uncertain. Moreover, the experimental eutectic temperature between U and UTe was overestimated in the measurements due to a chemical interaction between the sample and the crucible. Thus the discrepancy with the model was expected. New measurements shall be performed to improve the phase diagram knowledge in the $\mathrm{U}-\mathrm{UTe}_{2}$ composition range.

Concerning the thermodynamic data, the enthalpy of formation and standard entropies of the compounds are calculated in Figures 4 and 5, respectively.

The available data from the literature are reported for comparison. The model is in very good agreement with the experimental data of Baskin et al. [23] for $\mathrm{U}_{3} \mathrm{Te}_{4}$ whereas it is less good for UTe and $\mathrm{UTe}_{2}$. However, the value for $\mathrm{UTe}_{2}$ was not measured but estimated. Our calculated data are in good agreement with the data derived from the Calphad model of Wolf [20], except for UTe and $\mathrm{U}_{3} \mathrm{Te}_{4}$. Our data are much higher than the values coming from the Solgasmix model of Czechowitz [19]. Finally, as expected, our model is in disagreement with the DFT data which are very far from the experimental data of Baskin et al. [23]. Concerning the entropies, our model is in reasonable agreement with the estimations by Westrum [21] and the model of Wolf [20] who considered a mixing law between $U$ and Te. New measurements of thermodynamic data for the compounds would be very useful to improve the reliability of the model. 


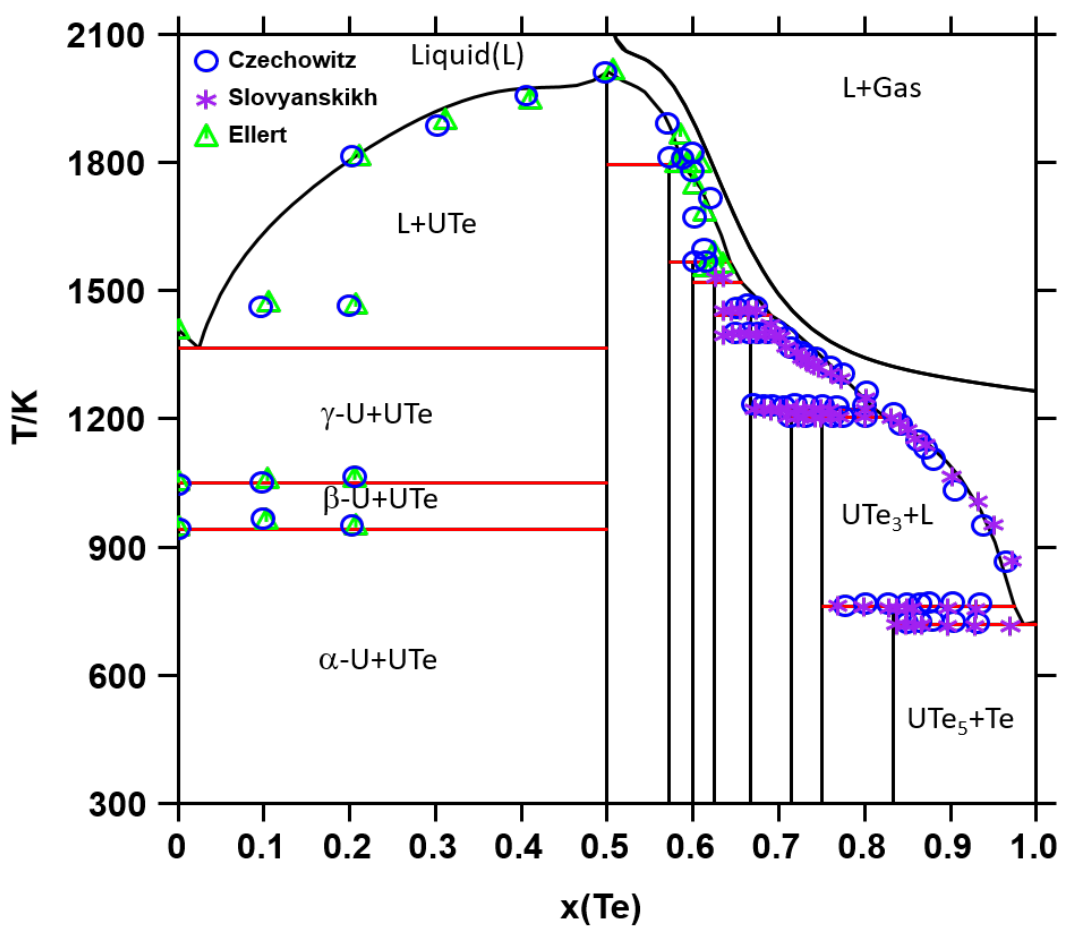

Figure 2. Calculated phase diagram. Comparison with the experimental data from Czechowitz [19], Slovyanskikh et al. [13] and Ellert et al. [18].

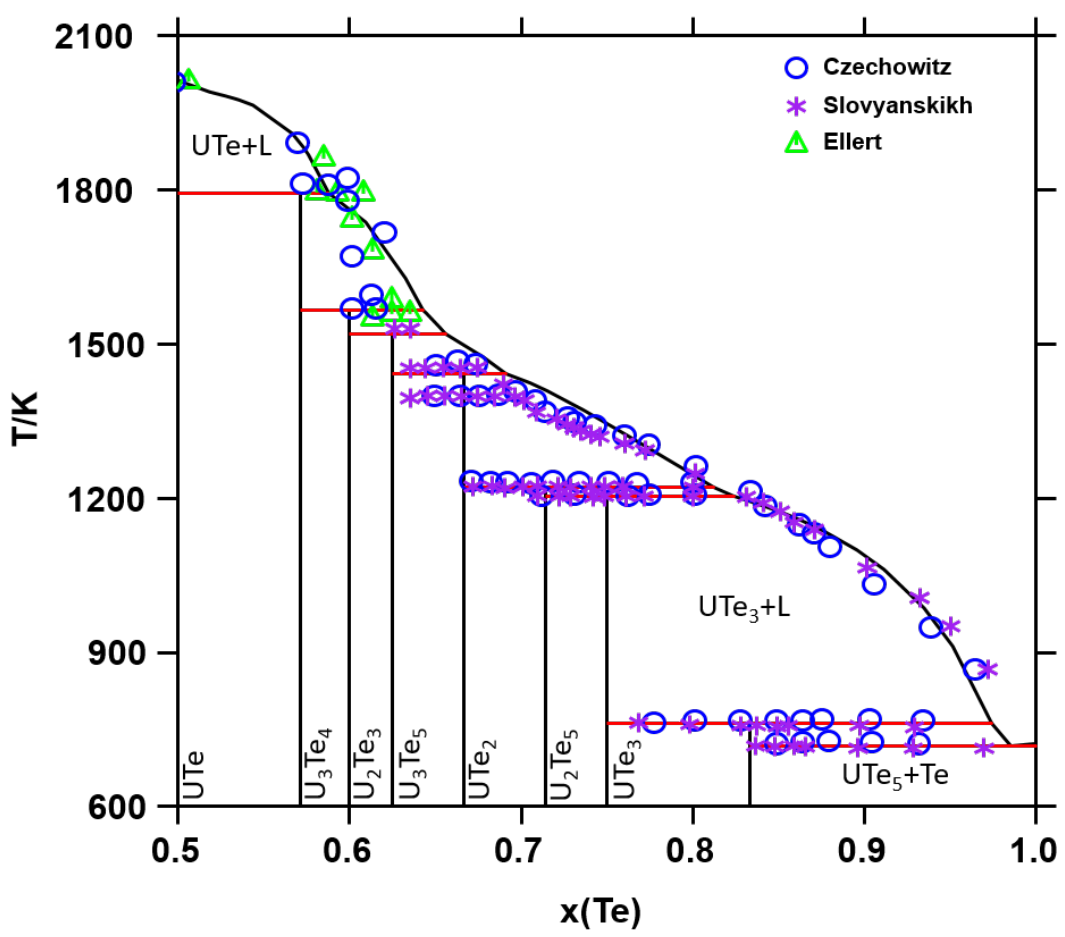

Figure 3. Calculated phase diagram from 50 to 100 at. \% Te. Comparison with the experimental data from Czechowitz [19], Slovyanskikh et al. [13] and Ellert et al. [18].

The calculated partial pressure data in the two phase regions (UTe $\left.+\mathrm{U}_{3} \mathrm{Te}_{4}\right),\left(\mathrm{U}_{3} \mathrm{Te}_{5}+\right.$ $\left.\mathrm{UTe}_{2}\right)$, and $\left(\mathrm{U}_{2} \mathrm{Te}_{5}+\mathrm{UTe}_{3}\right)$ are compared with the available experimental data in Figure 6. Our model is in very good agreement with the experimental data for the three two-phase regions. On the contrary, the data calculated with the model of Wolf [20] lead to a systematic underestimation of the partial pressure data. 
In conclusion, the CALPHAD model reproduces most of the selected data. However, the enthalpy of the formation of UTe is underestimated. This could be due to the inconsistency between the enthalpy of formation of UTe and the partial pressure data as already pointed out by Czechowitz [22].

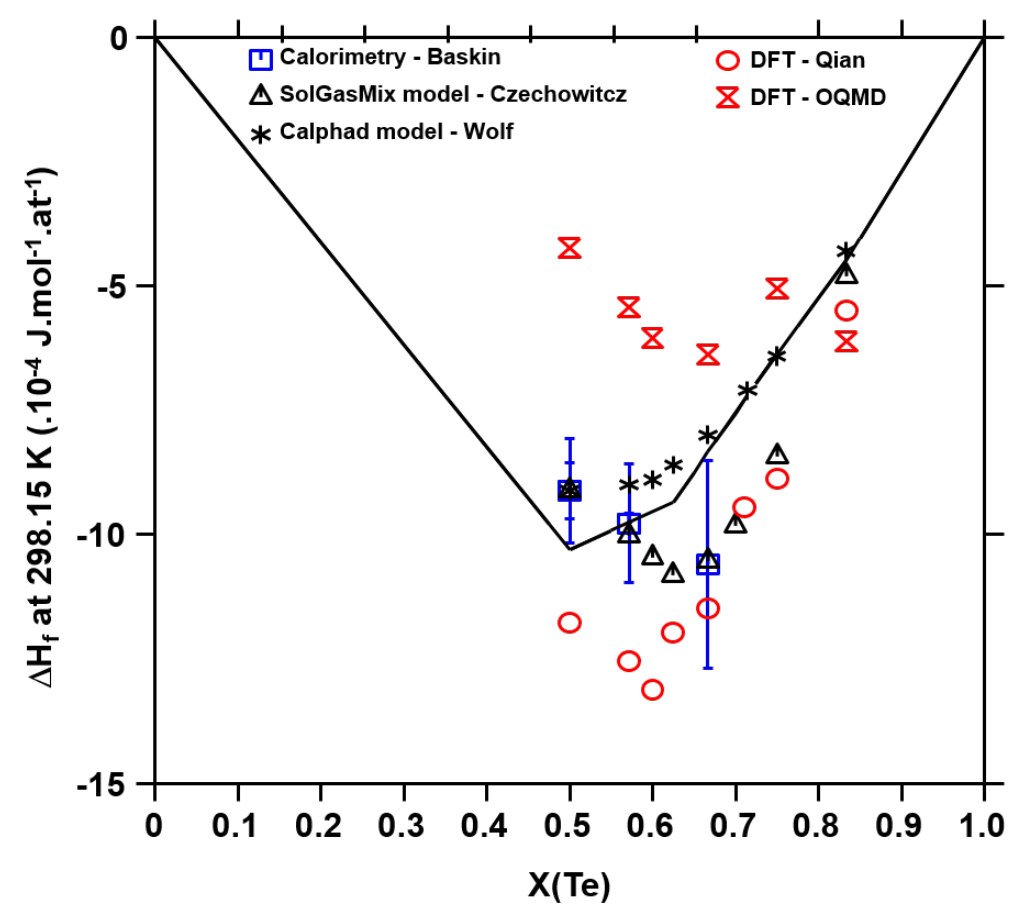

Figure 4. Calculated enthalpy of formation of $\mathrm{UTe}, \mathrm{U}_{3} \mathrm{Te}_{4}, \mathrm{U}_{2} \mathrm{Te}_{3}, \mathrm{U}_{3} \mathrm{Te}_{5}, \mathrm{UTe}_{2}, \mathrm{U}_{2} \mathrm{Te}_{5}, \mathrm{UTe}_{3}$, and $\mathrm{UTe}_{5}$. Comparison with the experimental data from Baskin et al. [23], the models from Czechowitz [19] and Wolf [20], and the DFT calculations from Qian [25] and the OQMD database [26].

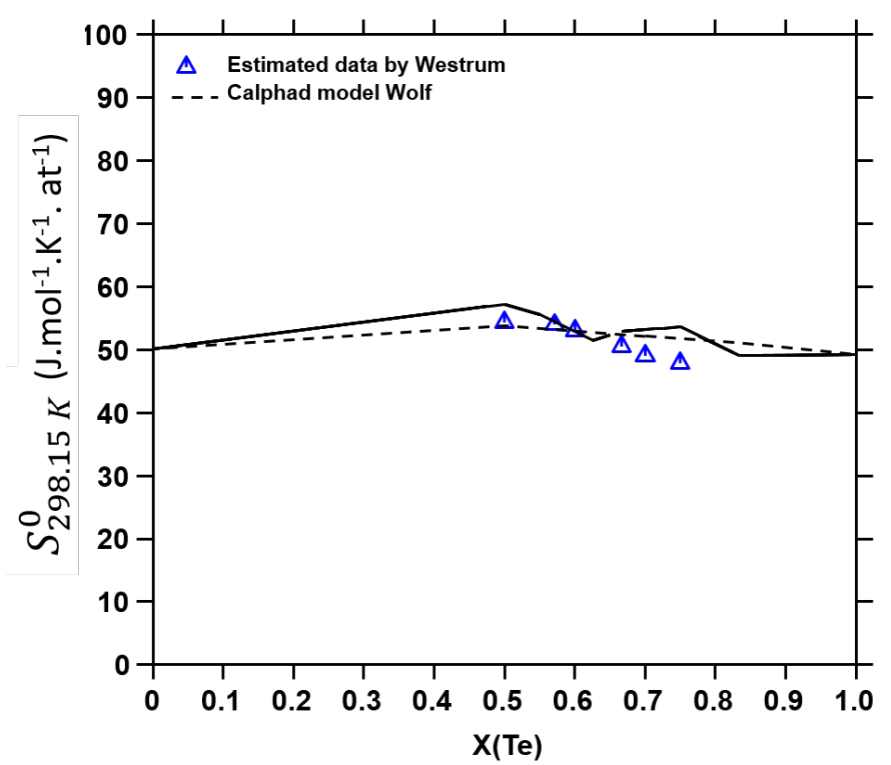

Figure 5. Calculated standard entropy at $298.15 \mathrm{~K}$ of $\mathrm{UTe}, \mathrm{U}_{3} \mathrm{Te}_{4}, \mathrm{U}_{2} \mathrm{Te}_{3}, \mathrm{UTe}_{2}, \mathrm{U}_{2} \mathrm{Te}_{5}$, and $\mathrm{UTe}_{3}$. Comparison with the estimated data from Westrum [21] and the model of Wolf [20].

To improve the reliability of the model, new measurements of the following data would be useful: Te activity, mixing enthalpy of formation in the liquid, enthalpy of formation, and heat capacity data for the compounds, phase diagram data in the $\mathrm{U}-\mathrm{UTe}_{2}$ region. 

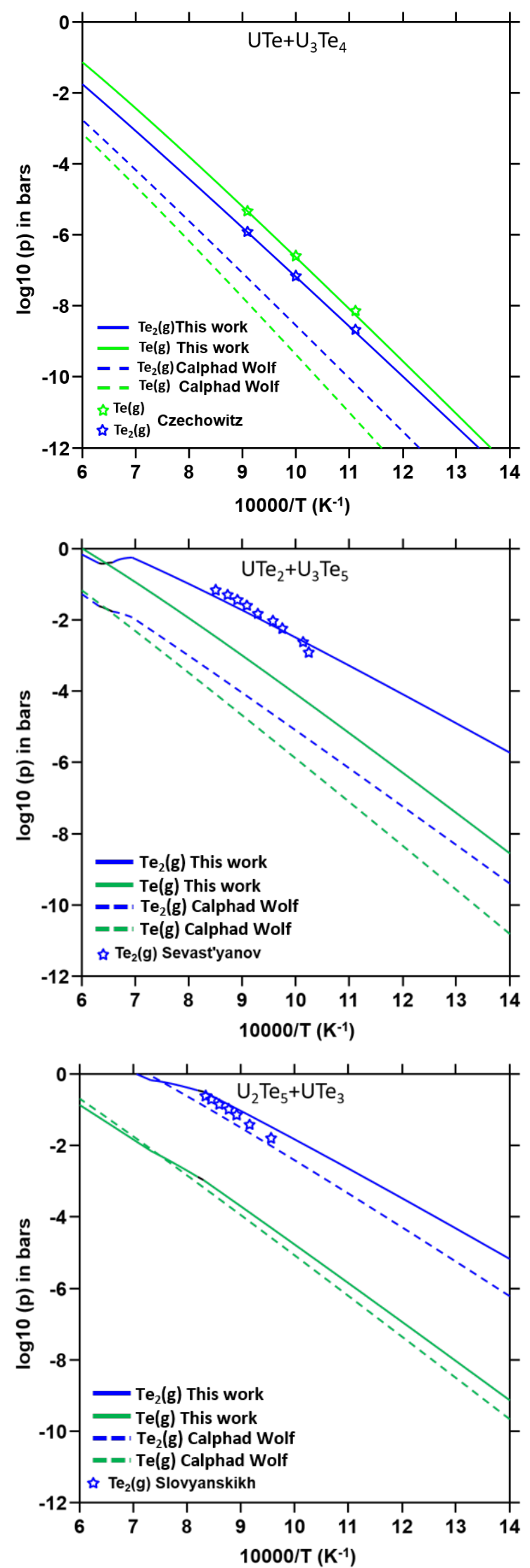

Figure 6. Calculated partial pressure of $\mathrm{Te}$ and $\mathrm{Te}_{2}$ in $\left(\mathrm{UTe}+\mathrm{U}_{3} \mathrm{Te}_{4}\right),\left(\mathrm{U}_{3} \mathrm{Te}_{5}+\mathrm{UTe}_{2}\right)$, and $\left(\mathrm{U}_{2} \mathrm{Te}_{5}\right.$ $\left.+\mathrm{UTe}_{3}\right)$ in plain lines. Comparison with the experimental results in $[22,27,28]$, and the model of Wolf [20] in dotted lines. 
Table 4. Posterior mean and standard deviation of the U-Te system parameters. The correlation matrix of the system parameters can be found in Appendix B.

\begin{tabular}{ccccc}
\hline Phase & Parameter Name & Assessment Value & Posterior Mean Value & Posterior Standard Deviation \\
\hline \multirow{4}{*}{ Liquid } & $L_{(U T e, T e)}^{0} B$ & $-171,903.278$ & $-171,903.269$ & 1.000 \\
& $L_{(U T e, T e)}^{0} C$ & 81.899 & 81.836 & 0.840 \\
& $L_{(U T e, T e)}^{1} B$ & $111,518.216$ & $111,518.211$ & 1.000 \\
& $L_{(U T e, T e)}^{1} C$ & -55.379 & -55.635 & 0.965 \\
& $L_{(U T e, T e)}^{2} B$ & $-69,986.365$ & $-69,986.364$ & 1.000 \\
& $L_{(U T e, T e)}^{2} C$ & 46.920 & 47.115 & 0.994 \\
\hline \multirow{2}{*}{$\mathrm{UTe}^{2}$} & $A_{0}$ & $-221,461.935$ & $-221,461.944$ & 1.000 \\
& $A_{1}$ & 198.439 & 198.314 & 0.236 \\
\hline \multirow{2}{*}{$\mathrm{U}_{3} \mathrm{Te}_{4}$} & $A_{0}$ & $-736,731.990$ & $-736,731.991$ & 1.000 \\
& $A_{1}$ & 689.583 & 689.274 & 0.478 \\
\hline \multirow{2}{*}{$\mathrm{U}_{2} \mathrm{Te}_{3}$} & $A_{0}$ & $-514,925.773$ & $-514,925.777$ & 1.000 \\
& $A_{1}$ & 494.721 & 494.781 & 0.379 \\
\hline \multirow{2}{*}{$\mathrm{U}_{3} \mathrm{Te}_{5}$} & $A_{0}$ & $-808,509.276$ & $-808,509.286$ & 1.000 \\
& $A_{1}$ & 796.312 & 796.470 & 0.686 \\
\hline \multirow{2}{*}{$\mathrm{UTe}_{2}$} & $A_{0}$ & $-272,474.416$ & $-272,474.408$ & 1.000 \\
& $A_{1}$ & 288.876 & 288.996 & 0.160 \\
\hline \multirow{2}{*}{$\mathrm{U}_{2} \mathrm{Te}_{5}$} & $A_{0}$ & $-556,197.559$ & $-556,197.562$ & 1.000 \\
& $A_{1}$ & 656.416 & 656.504 & 0.402 \\
\hline \multirow{2}{*}{$\mathrm{UTe}_{3}$} & $A_{0}$ & $-283,794.079$ & $-283,794.075$ & 1.000 \\
& $A_{1}$ & 367.797 & 367.730 & 0.250 \\
\hline \multirow{2}{*}{$\mathrm{UTe}_{5}$} & $A_{0}$ & $-312,579.609$ & $-312,579.607$ & 1.000 \\
& $A_{1}$ & 557.353 & 557.329 & 0.269 \\
\hline \multirow{2}{*}{} & & &
\end{tabular}

\subsection{Uncertainty Propagation with the Bayesian Approach}

In this section, the uncertainty propagation with the Bayesian approach is studied in the UTe-Te part of the phase diagram only, as there are too few data in the U-UTe part of the diagram to perform an uncertainty analysis. The final parameter values obtained in the assessment with PARROT as well as the posterior average and standard deviation values are reported in Table 4 .

Figures 7 and 8 show the plot matrices of the parameters posterior probability distribution: for the liquid phase and UTe in Figure 7 and for the solid compounds entropy parameters in Figure 8. Notice that it is possible to build such plot matrices for the whole set of parameters. However, for clarity purposes, small groups of parameters are selected. The groups selected are those showing more dependencies.

We briefly recall how to read such graphs. In Figure 8 the eight diagonal plots are the histograms of each entropy parameter. The scatterplots above the diagonal show the correlations between all parameters. For example in Figure 8, the first row gives the correlations between UTe and the other compounds. Ellipses and circular correlation scatterplots are a direct consequence of the Gaussian distribution. A thin ellipse means that there exists a strong relationship between the two parameters values. On the contrary, a circular scatterplot is the result of a low dependency.

Figure 8 shows strong correlations for the compounds that have close compositions. For example, the correlation values are above 0.97 for the pairs $\mathrm{U}_{2} \mathrm{Te}_{3}-\mathrm{U}_{3} \mathrm{Te}_{5}, \mathrm{U}_{2} \mathrm{Te}_{5}-\mathrm{UTe}_{3}$, and $\mathrm{UTe}_{3}-\mathrm{UTe}_{5}$. Conversely, the correlation values decrease as the compositions become more distant. The pair UTe-UTe 5 has the lowest correlation value, which is below 0.01 . The complete correlation matrix is given in the Appendix B. 
The other parameters are not very correlated. Figure 7 shows no correlation between the liquid parameters. The only strong correlation that can be observed on this graph is the one between the parameter $C$ for the order 0 interaction term and the entropy parameter for UTe with a correlation value slightly above -0.7 .

Using the methodology described in Section 4, the uncertainty of the different calculated thermodynamic quantities are compared with the experimental data in Figure 9 for $\Delta H_{f, 298.15 K}^{0}$, Figure 10 for $S_{298.15 K}^{0}$, and Figure 11 for the partial pressure corresponding to the two-phases regions $\left.\left(\mathrm{UTe}+\mathrm{U}_{3} \mathrm{Te}_{4}\right),\left(\mathrm{U}_{3} \mathrm{Te}_{5}+\mathrm{UTe}\right)_{2}\right)$, and $\left(\mathrm{U}_{2} \mathrm{Te}_{5}+\mathrm{UTe}_{3}\right)$. The prediction errors show a rather good agreement with the partial pressure data. The obtained credible intervals are in adequacy with the experimental results; circa plus or minus 0.1 bar for $\mathrm{UTe}-\mathrm{U}_{3} \mathrm{Te}_{4}$ and $\mathrm{UTe}_{2}-\mathrm{U}_{3} \mathrm{Te}_{5}$, plus or minus 0.05 bar for $\mathrm{UTe}_{3}-\mathrm{U}_{2} \mathrm{Te}_{5}$. In addition, for the partial pressure in the $\mathrm{UTe}-\mathrm{U}_{3} \mathrm{Te}_{4}$ region which shows the largest experimental uncertainty, the posterior credible interval length corresponds to half of the value of the experimental uncertainty. In Figures 9 and 10, the obtained propagated uncertainties appear to be rather small compared to the fitting data error bars and the obtained propagated uncertainty for the partial pressure in Figure 11. Such differences could be explained by the fact that the determination of partial pressure confidence sets involves several parameters whereas the confidence sets for $\Delta H_{f, 298.15 K}^{0}$ and $S_{298.15 K}^{0}$ depend on a single parameter. Both Figures 4 and 9 show the same trend and the same strong discrepancy for the $\Delta H_{f, 298.15 K}^{0}$ value of UTe. Despite such a gap with the thermodynamic data, the assessed model is in better agreement with the phase diagram.

In Figure 10, one can observe especially small credible intervals for UTe and $\mathrm{UTe}_{2}$. This observation is contrary to the expected result as very few fitting data were used. Considering the mismatch in the experimental data of $\Delta H_{f, 298.15 K}^{0}, S_{298.15 K}^{0}$ and the partial pressure, this unexpected result could be caused by inconsistent data. Meaning that it appears to be difficult to find parameters values that are in adequacy with all the fitting data.
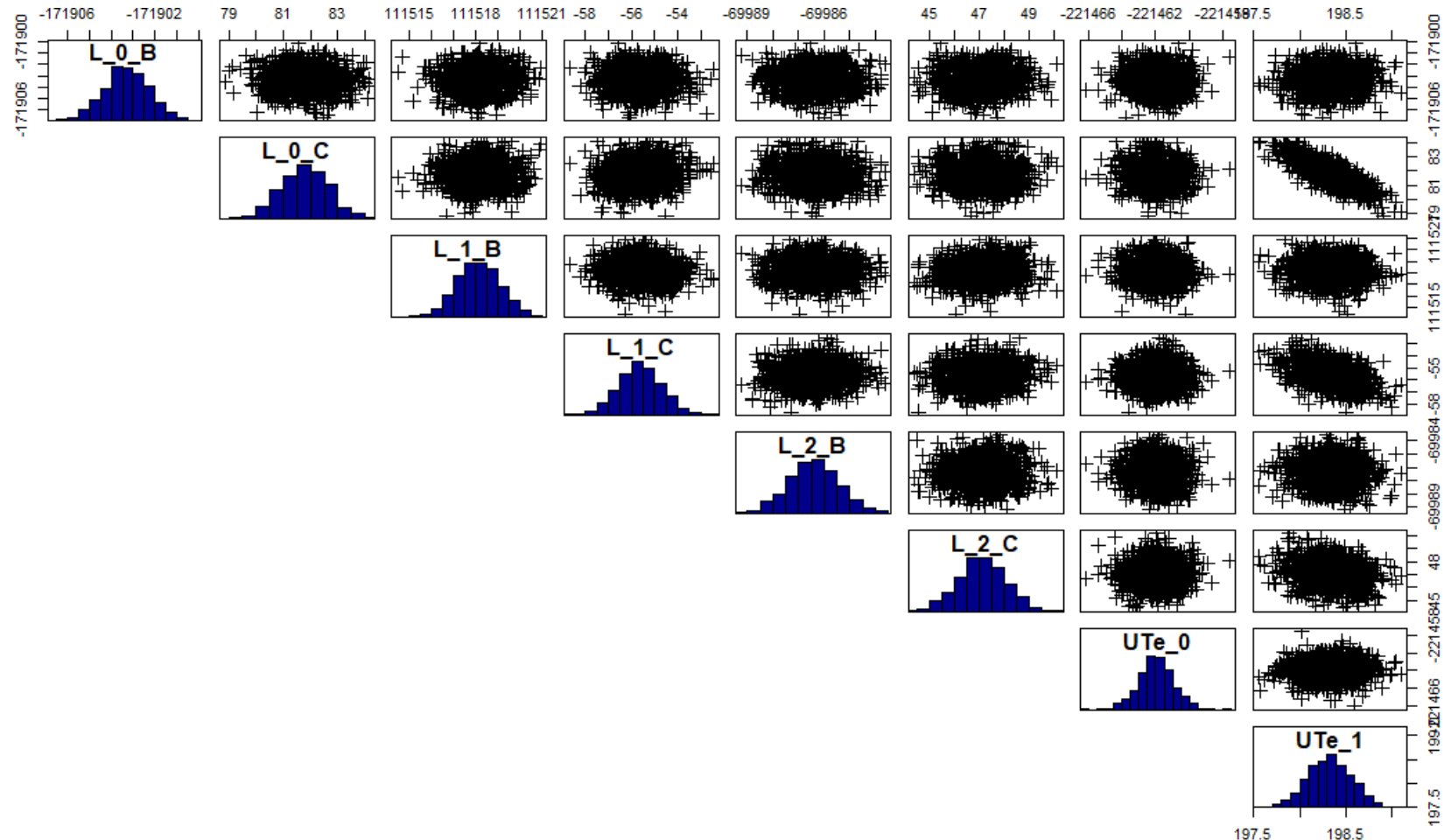

Figure 7. Parameter posterior probability distributions and correlations matrix plot for the liquid phase on UTe-Te side and UTe. The posterior correlation values can be found in Appendix B. 


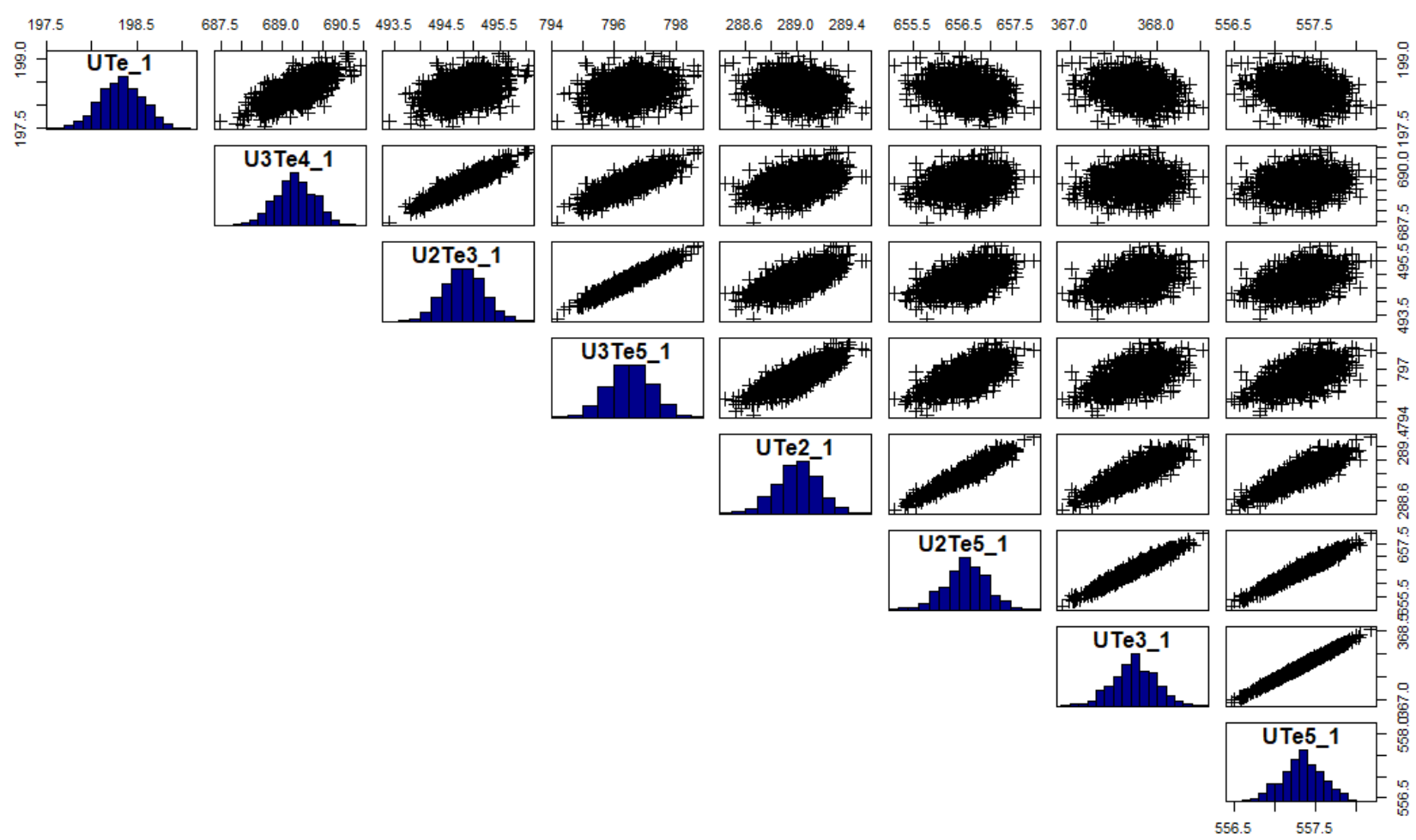

Figure 8. Parameter posterior probability distributions and correlations matrix plot for the compounds entropic parameters. The posterior correlation values can be found in Appendix B.

\section{Formation enthalpy per atom unit}

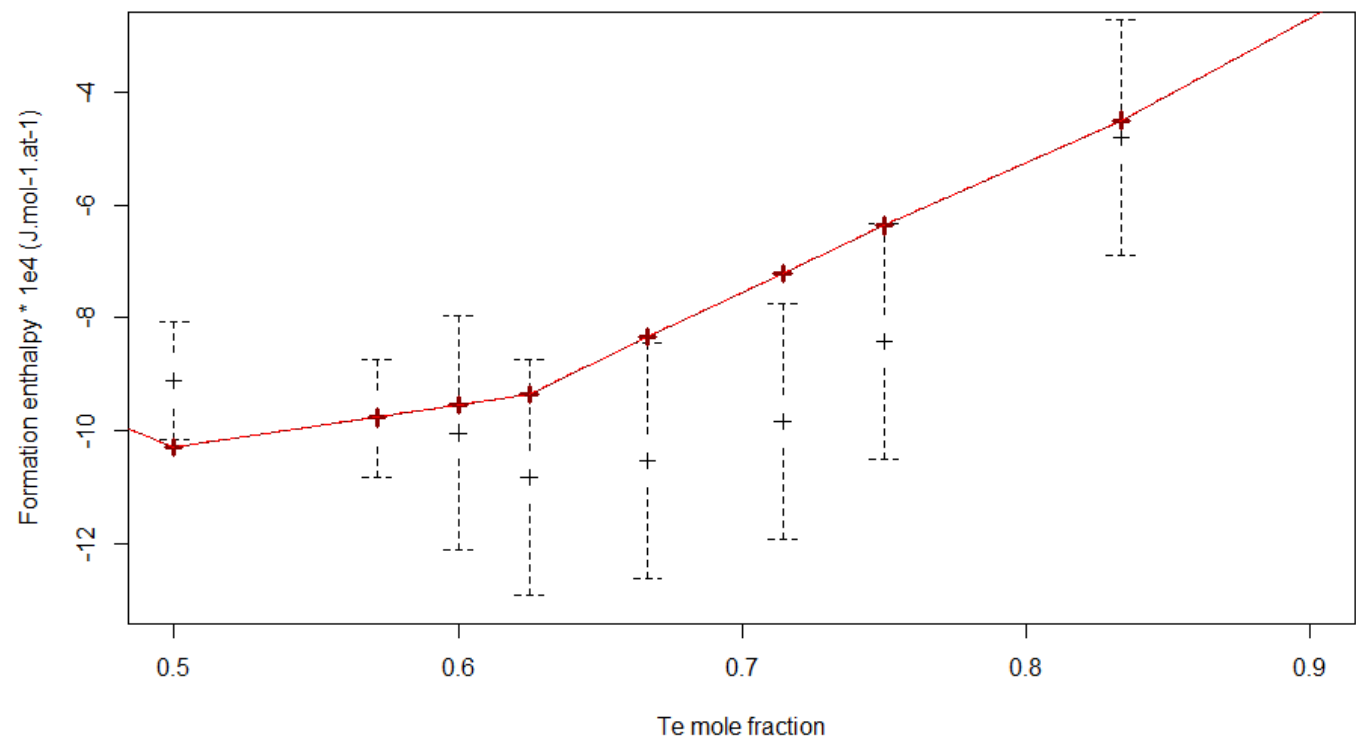

Figure 9. Uncertainty estimation given by the $95 \%$ credible interval of the posterior probability distribution for the calculated enthalpies of formation for $U T e, U_{3} T_{4}, U_{2} T_{3}, U_{3} T_{5}, U_{T}, U_{2} T_{5}$, $\mathrm{UTe}_{3}$, and $\mathrm{UTe}_{5}$. The experimental error bars are in dotted black lines. The propagated uncertainties (95\% credible interval) for each computed quantity are in bold red lines. 
Entropy per atom unit

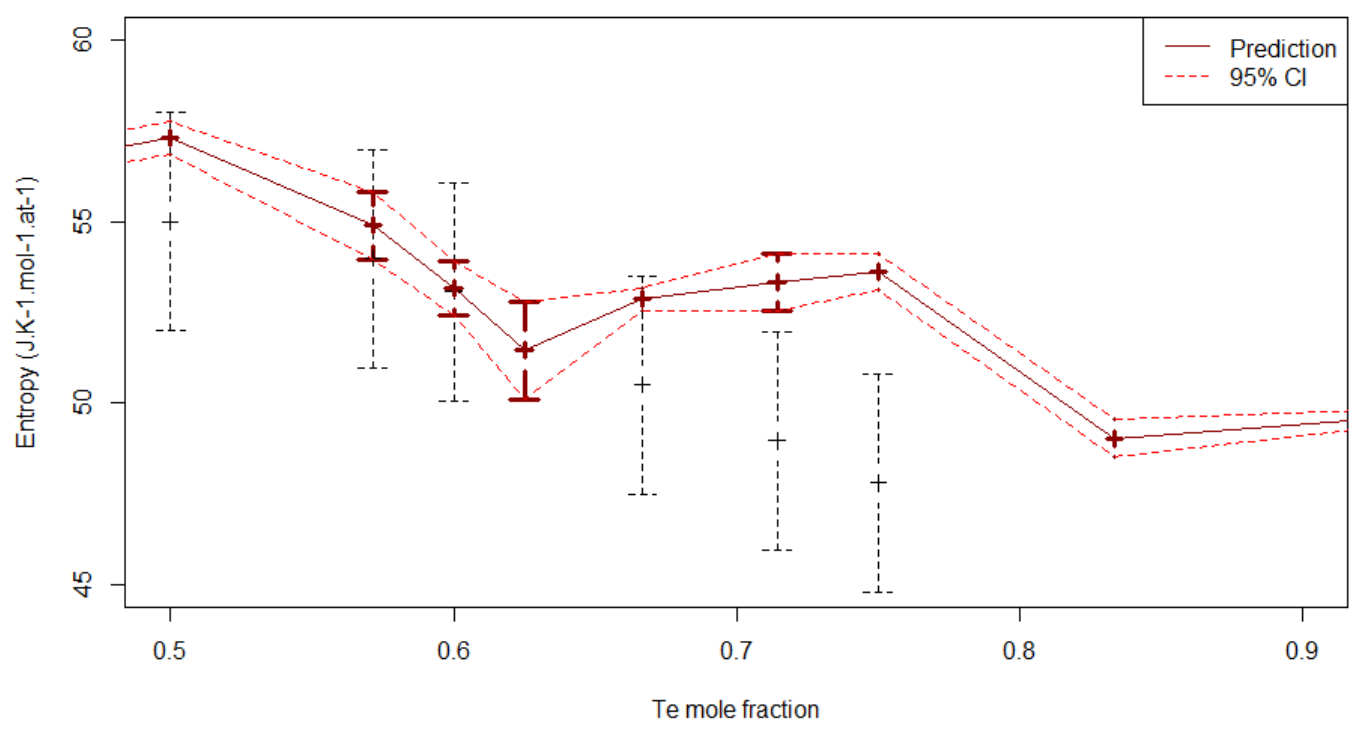

Figure 10. Uncertainty estimation given by the $95 \%$ credible interval of the posterior probability distribution for the calculated entropies for $\mathrm{UTe}, \mathrm{U}_{3} \mathrm{Te}_{4}, \mathrm{U}_{2} \mathrm{Te}_{3}, \mathrm{UTe}_{2}, \mathrm{U}_{2} \mathrm{Te}_{5}$, and $\mathrm{UTe}_{3}$. The experimental error bars are in doted black lines. The propagated uncertainties ( $95 \%$ credible interval) for each computed quantity are in bold red lines.

Figure 12 displays $N=100$ simulations of the phase diagram. Each phase diagram has been computed with the fifth version of the open-source software OpenCalphad [38,39]. Each simulation has been obtained by computing the phase diagram with a sample drawn from the posterior probability distribution of the whole set of parameters. Despite the small posterior uncertainty of the thermodynamic data, large posterior uncertainty can be observed on the right side of the diagram for the liquidus curve and the melting temperatures of $\mathrm{U}_{3} \mathrm{Te}_{4}, \mathrm{U}_{2} \mathrm{Te}_{3}, \mathrm{U}_{3} \mathrm{Te}_{5}, \mathrm{U}_{2} \mathrm{Te}_{5}$, and $\mathrm{UTe}_{3}$. The estimated uncertainty for the melting temperature of $\mathrm{U}_{2} \mathrm{Te}_{3}, \mathrm{U}_{3} \mathrm{Te}_{5}, \mathrm{U}_{2} \mathrm{Te}_{5}$, and $\mathrm{UTe}_{3}$ is around $50 \mathrm{~K}$ and $100 \mathrm{~K}$ for the melting temperature of $\mathrm{U}_{3} \mathrm{Te}_{4}$.

Notice that, for some parameters values drawn from the posterior probability distribution, the compounds $\mathrm{U}_{2} \mathrm{Te}_{3}, \mathrm{UTe}_{2}$, and $\mathrm{U}_{2} \mathrm{Te}_{5}$ may not be stable at $298.15 \mathrm{~K}$. Such results lead to the existence of an invariant transition below $350 \mathrm{~K}$ for these compounds. In the phase diagrams found in the literature, these compounds are assumed to be stable at room temperature $[9,13,18,19]$. However, more enthalpy of formation data are required to confirm this assumption. Moreover, these compounds have very close compositions, and even small variation of parameters values can lead to unstable compounds at room temperature. 
UTe + U3Te4

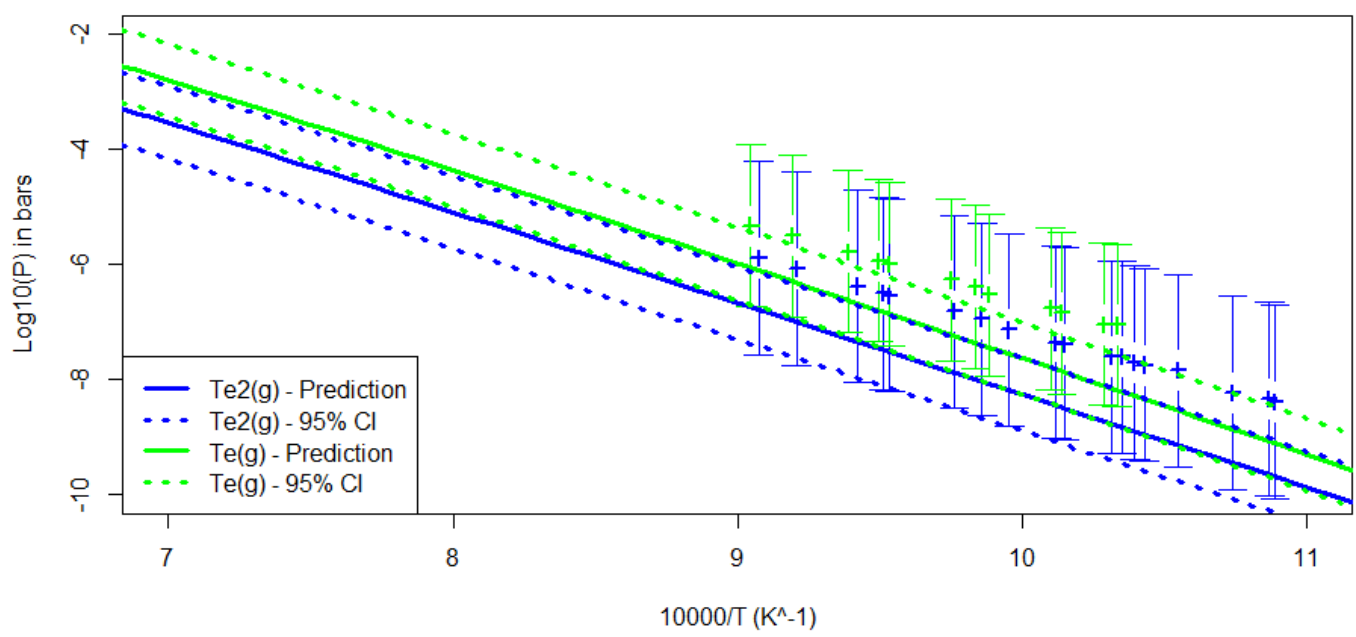

U3Te5 + UTe2
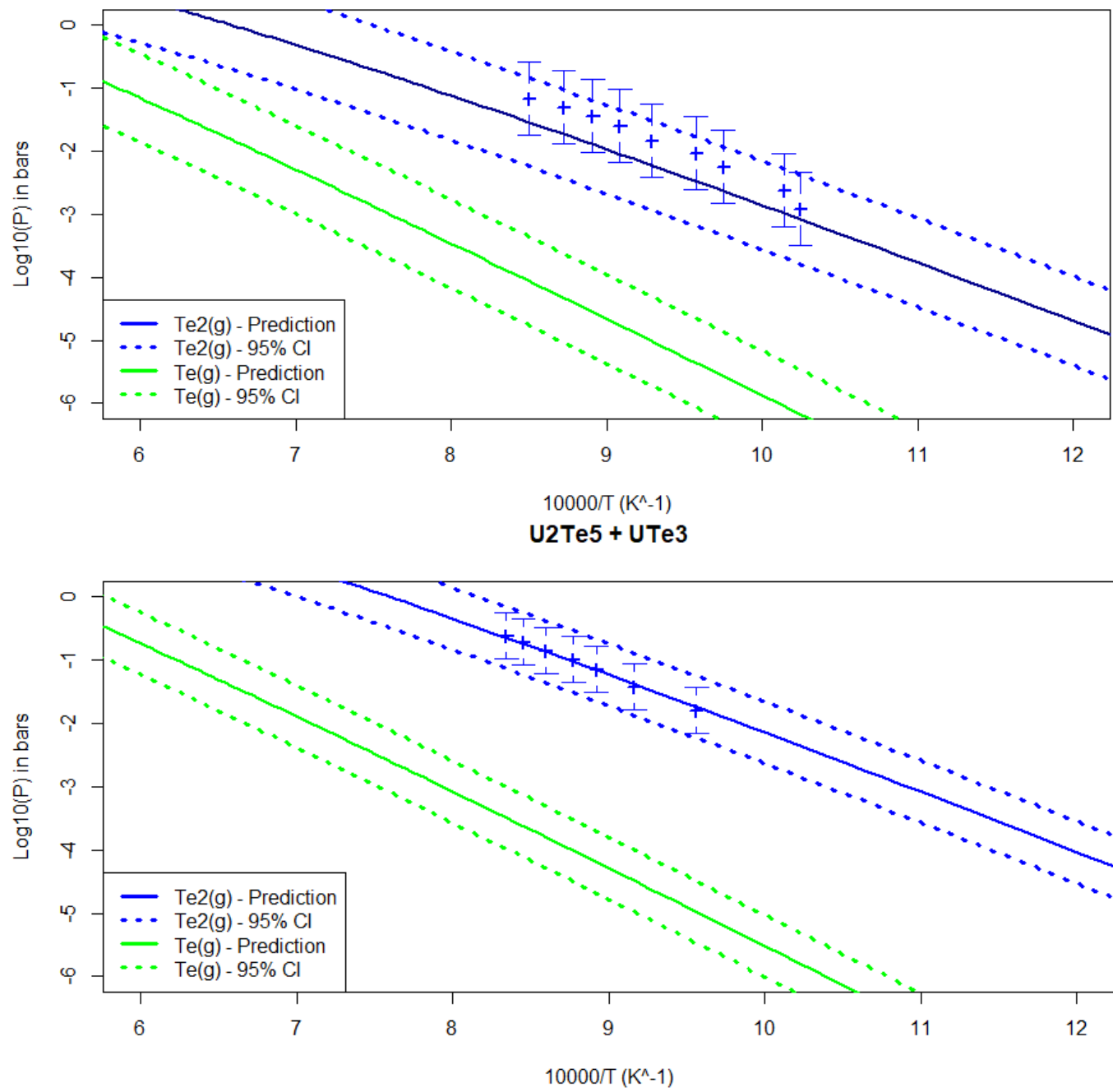

Figure 11. Uncertainty estimation given by the $95 \%$ credible interval of the posterior probability distribution for the calculated partial pressure. Plain lines are the posterior means. Dotted lines correspond to the $95 \%$ upper and lower bounds of the credible interval for each temperature value. 


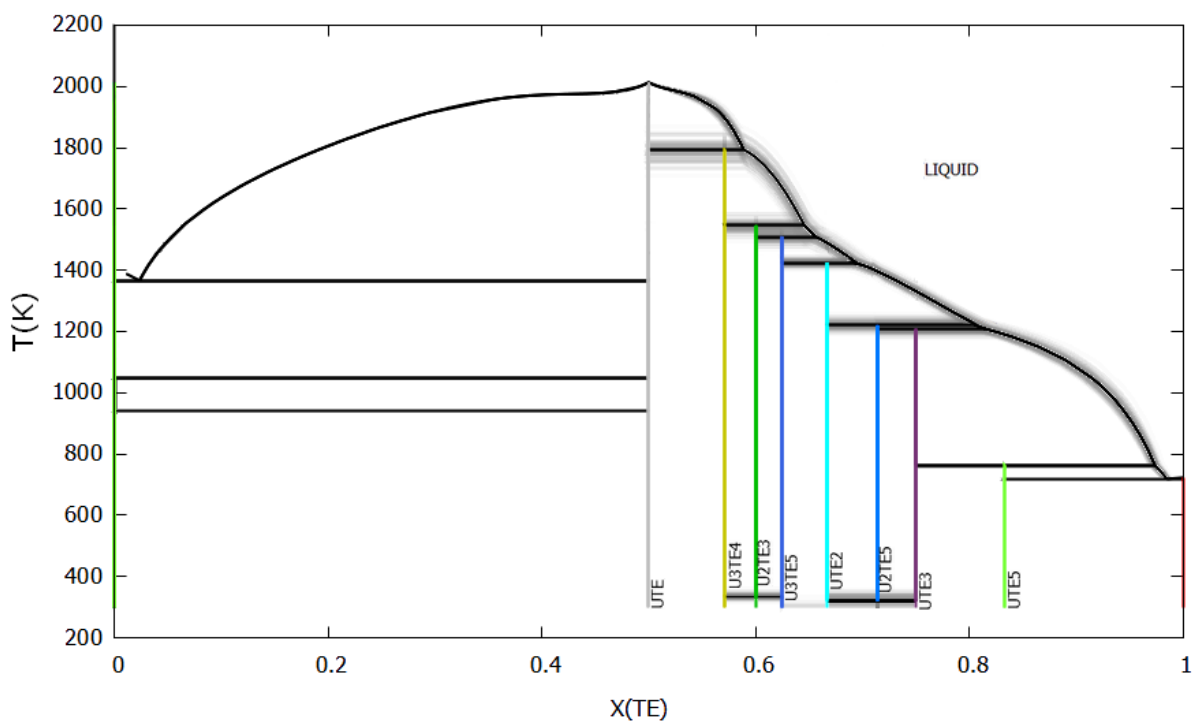

Figure 12. Simulation of $N=100$ phase diagram from the parameters posterior probability distribution.

\section{Conclusions}

In a first step, the thermodynamic assessment of the uranium-tellurium system has been performed using the CALPHAD method. Experimental phase diagram and thermodynamic data have been used as input data to optimize the Gibbs energy functions for UTe, $\mathrm{U}_{3} \mathrm{Te}_{4}, \mathrm{U}_{2} \mathrm{Te}_{3}, \mathrm{U}_{3} \mathrm{Te}_{5}, \mathrm{UTe}_{2}, \mathrm{U}_{2} \mathrm{Te}_{5}, \mathrm{UTe}_{3}$, and $\mathrm{UTe}_{5}$ and for the liquid phase.

It should be noted that there do not exist any thermodynamic data on the liquid phase. Moreover, for the compounds, measurements of enthalpy of formation data at $298 \mathrm{~K}$ are only available for $\mathrm{UTe}$ and $\mathrm{U}_{3} \mathrm{Te}_{4}$, for which large error bars were assigned due to the large uncertainties in the measurements. Only estimations exist for the other compounds and all the enthalpy data show very large discrepancies. Unfortunately, the DFT calculations, which are very tricky on uranium tellurium compounds, did not help to make a choice as the results are far from the few experimental existing data.

For the standard entropy data at $298 \mathrm{~K}$, only estimations are available. Due to the absence of experimental data, the Kopp-Neumann law was used for the heat capacity function for the compounds. The only other experimental data available are vapor pressure measurements in two phase regions: $\mathrm{UTe}-\mathrm{U}_{3} \mathrm{Te}_{4}, \mathrm{UTe}_{2}-\mathrm{U}_{3} \mathrm{Te}_{5}$, and $\mathrm{U}_{2} \mathrm{Te}_{5}-\mathrm{UTe} \mathrm{T}_{3}$. Input thermodynamic data for the model were carefully selected. The phase diagram from pure $\mathrm{U}$ to $\mathrm{UTe}_{2}$ is quite uncertain. In fact, only a few liquidus data were measured. On the contrary, the phase diagram is very well established from $\mathrm{UTe}_{2}$ to pure Te.

The CALPHAD model reproduces very well the experimental vapor pressure data and enthalpy of formation for $\mathrm{U}_{3} \mathrm{Te}_{4}$. However, the calculated enthalpy of formation for UTe is underestimated by $13 \%$ compared to the measured value. The agreement with Westrum estimations for the entropies of $\mathrm{U}_{3} \mathrm{Te}_{4}$ and $\mathrm{U}_{2} \mathrm{Te}_{3}$ is very good. Higher values are obtained for the other compounds with deviations from $5 \%$ (for $\mathrm{UTe}, \mathrm{UTe}_{2}$ ) to $12 \%$ for $\mathrm{UTe}_{3}$. The calculated phase diagram is in very good agreement with the experimental data. For comparison, the CALPHAD model of Wolf leads to a very good agreement with the enthalpy of formation for UTe whereas it overestimates the enthalpy for $\mathrm{U}_{3} \mathrm{Te}_{4}$. Moreover, it tends to underestimate systematically the vapor pressure data and the liquidus curve are not well reproduced compared to the experimental points. It clearly shows that in both CALPHAD assessments, it was finally not possible to get a good agreement with all the experimental data. One of the reasons is the inconsistency between the experimental enthalpy of formation data and the vapor pressure measurements.

In a second step, the uncertainty analysis was performed on the CALPHAD model. The prediction error shows a good agreement with the partial pressure experimental uncertainty and small confidence intervals. The prediction model shows more discrepancies 
with the entropy data and the enthalpy of formation data. The prediction error however remains very small (less than $0.1 \%$ ) for the enthalpy of formation and quite small (between $1 \%$ and $4 \%$ ) for the entropy.

Despite the global little uncertainty on the predicted thermodynamic data, one can observe rather large estimated uncertainty on the phase diagram. In particular the estimated uncertainty for the melting temperature of $\mathrm{U}_{3} \mathrm{Te}_{4}$ is around $100 \mathrm{~K}$.

From a global perspective, the whole system lacks a sufficient amount of fitting data. The system complexity and the number of parameters are too important for the too few numbers of fitting data (120 points versus 22 parameters) and we do not have any thermodynamic data on the liquid. New experimental data, like heat capacity, enthalpy of formation measurements for the different compounds, and chemical potential values for the liquid phase could improve the model.

Given our results, we have little confidence in the assessed model and recommend additional measurements to improve the knowledge on the UTe-Te side of the system.

From a wider perspective, we renew with this study the advantages of the conjugate prior approach. Contrary to other Bayesian methods, such an approach allows us to have an analytical posterior probability distribution and, therefore, closed-form expressions for the uncertainty of the calculated thermodynamic quantities. Having an analytical posterior probability distribution also speeds up the simulation of the system parameters which makes easier the study of the uncertainty propagation to the phase diagram. In further work, we will apply this methodology to the study of a ternary system and to the propagation of the system parameters uncertainty when coupling with a multi-physics code.

Author Contributions: Conceptualization, C.G.; Data curation, C.G.; Investigation, C.G., E.L., T.K. and F.G.; Methodology, E.L.; Writing-original draft, C.G. and E.L.; Writing-review \& editing, C.G., E.L., T.K. and F.G. All authors have read and agreed to the published version of the manuscript.

Funding: This work was supported by the Cross-Cutting basic research Program (RTA Program) of the CEA Energy Division. This research is part of the INSPYRE project which has received funding from the Euratom research and training program 2014-2018 under Grant Agreement No 754329. This research contributes to the joint program on nuclear materials (JPNM) of the European energy research alliance (EERA).

Institutional Review Board Statement: Not applicable.

Informed Consent Statement: Not applicable.

Data Availability Statement: Not applicable.

Acknowledgments: Support from the ANR-3IA Artificial and Natural Intelligence Toulouse Institute is gratefully acknowledged. The authors express their acknowledgements to Bo Sundman, Philippe Zeller and Jean-Louis Flèche for helpful technical discussions.

Conflicts of Interest: The authors declare no conflict of interest.

\section{Appendix A. Reduction of the Number of Parameters}

\section{Appendix A.1. Degree of Freedom and System Dimension Reduction}

The system modeling proposed in this work features 22 parameters ( 6 interactions of UTe-Te parameters for the liquid and 2 parameters for each of the 8 solid compounds).

The degree of freedom for the thermodynamic data is equal to 15 . This value is obtained as follows. There are 8 observations for the enthalpy values (one for each compound) and 6 observations for the entropy values (no values for $\mathrm{U}_{3} \mathrm{Te}_{5}$ and $\mathrm{UTe}_{5}$ ). The partial pressure observations give linear relations between the enthalpy and the entropy parameters for compounds involved in the reaction. One degree of freedom (corresponding to the entropic parameter of $\mathrm{U}_{3} \mathrm{Te}_{5}$ ) is added due to these partial pressure relations.

No information is provided on the $\mathrm{UTe}_{5}$ entropic term and the liquid parameters by the thermodynamic data. The use of the invariant transitions is necessary to determine the entire set of parameters probability distributions. Such invariant transitions are the melting 
of compounds $\mathrm{U}_{3} \mathrm{Te}_{4}, \mathrm{U}_{2} \mathrm{Te}_{3}, \mathrm{U}_{3} \mathrm{Te}_{5}, \mathrm{UTe}_{2}, \mathrm{U}_{2} \mathrm{Te}_{5}, \mathrm{UTe}_{3}$, and $\mathrm{UTe}_{5}$. The melting of $\mathrm{U}_{3} \mathrm{Te}_{4}$ involves liquid parameters and $\mathrm{UTe}$ and $\mathrm{U}_{3} \mathrm{Te}_{4}$ parameters. The other melting conditions involve 4 compounds parameters, the UTe parameters, and the liquid parameters.

The 7 melting conditions involve the $\mathrm{UTe}_{5}$ entropic parameter and the liquid parameters which give sufficient conditions to identify the parameters probability distributions.

\section{Appendix A.2. Reduced Prior Probability Distribution and Relations with the Initial Set of Parameters}

In this section, $p$ denotes the number of system parameters, $k$ is the degree of freedom for the thermodynamic data and $h$ is the number of invariant transitions (melting conditions). In the U-Te system, we have $p=22, k=15$ and $h=7$. Notice that $p=k+h$ is a necessary condition for the study. We denote by $\theta$ the $\mathbb{R}^{p}$-vector featuring the vector of system parameters.

More formally, the invariant transition conditions can be written as the following equation to be satisfied by the parameter $\theta$

$$
M \theta=c
$$

where $M \in \mathbb{R}^{h \times p}$ and $c \in \mathbb{R}^{h}$.

We denote by ker $M$ the subset of vectors $u \in \mathbb{R}^{p}$ that are such that $M u=0$. Provided that it exists $\theta_{0} \in \mathbb{R}^{p}$ that is such that

$$
M \theta_{0}=c
$$

then, for all $u \in \operatorname{ker} M$, all vectors defined as $u+\theta_{0}$ satisfy (A1).

Let $k$ be the dimension of ker $M$. Let $P \in \mathbb{R}^{p \times k}$ be the matrix formed by the $k$ eigen vectors in columns of $M$ related to the eigen value 0 . Then all the random vectors generated by the prior probability distribution $\mathcal{N}_{p}\left(\theta_{0}, P P^{t}\right)$ satisfy the conditions (A1). Notice that $P P^{t}$ is a $p \times p$ matrix with rank $\left(P P^{t}\right)=k$. Therefore, we can generate the random vectors in a reduced subspace of dimension $k$.

In this reduced subspace, the prior probability distribution is the Gaussian standard $\mathcal{N}_{k}\left(0, I_{k}\right)$. Then, the posterior probability distribution is the Gaussian probability distribution $\mathcal{N}_{k}\left(v_{k}, s_{k}\right)$ with

$$
\begin{aligned}
& s_{k}=\left(P^{t} D^{t} s_{Z}^{-1} D P+I_{k}\right)^{-1} \\
& v_{k}=s_{k} P^{t} D^{t} s_{Z}^{-1} Z
\end{aligned}
$$

where $D$ is the design matrix corresponding to the thermodynamic observations $Z$ (enthalpy, entropy, and partial pressures data) and $s_{Z}$ are the observations uncertainties.

The posterior probability distribution in the initial space of parameters can be determined given the relation

$$
\theta=P v+\theta_{0} .
$$

Therefore the posterior probability distribution in $\mathbb{R}^{p}$ is the Gaussian distribution $\mathcal{N}_{p}\left(\theta_{p}, s_{p}\right)$ with

$$
\begin{aligned}
& s_{p}=P\left(P^{t} D^{t} s_{Z}^{-1} D P+I_{k}\right)^{-1} P^{t} \\
& \theta_{p}=P s_{k} P^{t} D^{t} s_{Z}^{-1} Z+\theta_{0} .
\end{aligned}
$$

Finally, the value calculated by the assessment with Thermocalc is chosen as the value $\theta_{0}$ of condition (A2). 


\section{Appendix B. Additional Table: Correlation Values for the Posterior Probability Distribution}

Table A1. Posterior correlation matrix.

\begin{tabular}{|c|c|c|c|c|c|c|c|c|c|c|c|c|}
\hline & $L_{0} B$ & $L_{0} C$ & $L_{1} B$ & $L_{1} C$ & $L_{2} B$ & $L_{2} C$ & $u T e_{0}$ & $u T e_{1}$ & U3Te $4_{0}$ & U3Te $4_{1}$ & $U 2 \mathrm{Te} 3_{0}$ & $U 2 T e 3_{1}$ \\
\hline$L_{0} B$ & 1 & -0.015 & -0.010 & -0.012 & -0.046 & 0.030 & 0.034 & 0.028 & -0.010 & 0.034 & 0.052 & 0.031 \\
\hline$L_{0} C$ & & 1 & -0.009 & 0.036 & -0.078 & -0.033 & -0.002 & -0.688 & -0.012 & -0.368 & -0.018 & -0.100 \\
\hline$L_{1} B$ & & & 1 & 0.021 & -0.006 & 0.002 & 0.039 & -0.007 & 0.020 & -0.006 & 0.055 & -0.006 \\
\hline$L_{1} C$ & & & & 1 & -0.033 & -0.040 & 0.018 & -0.296 & -0.024 & -0.002 & 0.061 & 0.137 \\
\hline$L_{2} B$ & & & & & 1 & 0.012 & 0.074 & 0.063 & 0.055 & 0.032 & -0.018 & 0.008 \\
\hline$L_{2} \mathrm{C}$ & & & & & & 1 & 0.030 & -0.143 & 0.019 & 0.013 & -0.010 & 0.047 \\
\hline$U T e_{0}$ & & & & & & & 1 & -0.014 & 0.036 & -0.003 & 0.020 & 0.000 \\
\hline$U T e_{1}$ & & & & & & & & 1 & 0.008 & 0.854 & -0.010 & 0.661 \\
\hline$U 3 \mathrm{Te} 4_{0}$ & & & & & & & & & 1 & -0.002 & -0.011 & -0.006 \\
\hline U3Te4 1 & & & & & & & & & & 1 & -0.003 & 0.953 \\
\hline$U 2 \mathrm{Te} 3_{0}$ & & & & & & & & & & & 1 & -0.003 \\
\hline \multirow[t]{2}{*}{$U 2 \mathrm{Te}_{1}$} & & & & & & & & & & & & 1 \\
\hline & $U 3 T e 5_{0}$ & $U 3 T e 5_{1}$ & $U T e 2_{0}$ & $U T e 2_{1}$ & $U 2 T e 5_{0}$ & $U 2 T e 5_{1}$ & $U T e 3_{0}$ & $u T e 3_{1}$ & $U T e 5_{0}$ & $U T e 5_{1}$ & & \\
\hline$L_{0} B$ & 0.020 & 0.027 & -0.015 & 0.017 & -0.044 & 0.016 & -0.018 & 0.014 & 0.020 & 0.017 & & \\
\hline$L_{0} C$ & -0.011 & 0.121 & -0.034 & 0.463 & 0.021 & 0.615 & -0.007 & 0.687 & 0.004 & 0.682 & & \\
\hline$L_{1} B$ & 0.001 & -0.008 & 0.037 & -0.012 & 0.005 & -0.017 & -0.014 & -0.020 & 0.027 & -0.022 & & \\
\hline$L_{1} C$ & 0.010 & 0.147 & -0.023 & 0.093 & 0.017 & -0.133 & -0.055 & -0.292 & 0.022 & -0.389 & & \\
\hline$L_{2} B$ & 0.015 & -0.010 & 0.013 & -0.036 & -0.031 & -0.041 & -0.011 & -0.041 & -0.009 & -0.037 & & \\
\hline$L_{2} \mathrm{C}$ & 0.020 & -0.001 & -0.015 & -0.094 & 0.001 & -0.052 & 0.038 & -0.009 & 0.010 & 0.104 & & \\
\hline$U T e_{0}$ & -0.008 & -0.002 & 0.013 & -0.005 & 0.035 & -0.008 & -0.004 & -0.009 & 0.083 & -0.008 & & \\
\hline$U T e_{1}$ & 0.030 & 0.514 & 0.067 & 0.245 & -0.055 & 0.126 & 0.010 & 0.037 & 0.003 & 0.008 & & \\
\hline$U 3 \mathrm{Te} 4_{0}$ & -0.009 & -0.010 & 0.003 & -0.013 & -0.005 & -0.008 & -0.006 & -0.004 & -0.009 & 0.000 & & \\
\hline U3Te4 $4_{1}$ & 0.045 & 0.870 & 0.063 & 0.648 & -0.059 & 0.489 & -0.003 & 0.351 & 0.018 & 0.295 & & \\
\hline$U 2 \mathrm{Te} 3_{0}$ & -0.020 & -0.004 & -0.020 & -0.013 & -0.036 & -0.029 & 0.037 & -0.039 & 0.001 & -0.045 & & \\
\hline$U 2 \mathrm{Te}_{1}$ & 0.047 & 0.975 & 0.053 & 0.826 & -0.054 & 0.674 & -0.011 & 0.530 & 0.023 & 0.462 & & \\
\hline$U 3 T e 5_{0}$ & 1 & 0.043 & -0.039 & 0.034 & 0.002 & 0.025 & 0.040 & 0.018 & 0.021 & 0.017 & & \\
\hline$U 3 T e 5_{1}$ & & 1 & 0.046 & 0.931 & -0.049 & 0.810 & -0.014 & 0.680 & 0.024 & 0.606 & & \\
\hline$U T e 2_{0}$ & & & 1 & 0.029 & -0.009 & 0.026 & 0.003 & 0.020 & -0.016 & 0.017 & & \\
\hline$U T e 2_{1}$ & & & & 1 & -0.038 & 0.955 & -0.015 & 0.869 & 0.021 & 0.797 & & \\
\hline$U 2 T e 5_{0}$ & & & & & 1 & -0.034 & -0.018 & -0.027 & 0.033 & -0.025 & & \\
\hline$U 2 T e 5_{1}$ & & & & & & 1 & -0.001 & 0.977 & 0.014 & 0.937 & & \\
\hline$U T e 3_{0}$ & & & & & & & 1 & 0.006 & -0.070 & 0.021 & & \\
\hline $\mathrm{UTe}_{1}$ & & & & & & & & 1 & 0.009 & 0.987 & & \\
\hline$U T e 5_{0}$ & & & & & & & & & 1 & 0.003 & & \\
\hline$U T e 5_{1}$ & & & & & & & & & & 1 & & \\
\hline
\end{tabular}

\section{References}

1. Samuelsson, K.; Dumas, J.C.; Sundman, B.; Lainet, M. An improved method to evaluate the "Joint Oxyde-Gaine" formation in $(\mathrm{U}, \mathrm{Pu}) \mathrm{O}_{2}$ irradiated fuels using the GERMINAL V2 code coupled to Calphad thermodynamic computations. EPJ Nucl. Sci. Technol. 2020, 6, 47. [CrossRef]

2. Samuelsson, K.; Dumas, J.C.; Sundman, B.; Lamontagne, J.; Guéneau, C. Simulation of the chemical state of high burnup (U,Pu)O ${ }_{2}$ fuel in fast reactors based on thermodynamic calculations. J. Nucl. Mater. 2020, 532, 151969. [CrossRef]

3. Guéneau, C.; Dupin, N.; Kjellqvist, L.; Geiger, E.; Kurata, M.; Gossé, S.; Corcoran, E.; Quaini, A.; Hania, R.; Smith, A.L.; et al. TAF-ID: An international thermodynamic database for nuclear fuels applications. Calphad 2021, 72, 102212. [CrossRef]

4. Lukas, H.L.; Fries, S.G.; Sundman, B. Computational Thermodynamics: The Calphad Method; Cambridge University Press: Cambridge, UK, 2007.

5. Paulson, N.H.; Bocklund, B.J.; Otis, R.A.; Liu, Z.-K.; Stan, M. Quantified uncertainty in thermodynamic modeling for materials design. Acta Mater. 2019, 174, 9-15. [CrossRef]

6. Paulson, N.H.; Jennings, E.; Stan, M. Bayesian strategies for uncertainty quantification of the thermodynamic properties of materials. Int. J. Eng. Sci. 2019, 142, 74-93. [CrossRef]

7. Paulson N.H.; Zomorodpoosh, S.; Roslyakova, I.; Stan, M. Comparison of statistically-based methods for automated weighting of experimental data in CALPHAD-type assessment. Calphad 2020, 68, 101728. [CrossRef] 
8. Thermo-Calc Software, Version 2021a. Available online: https://thermocalc.com/ (accessed on 15 January 2021).

9. Okamoto, H. Te-U (Tellurium-Uranium). J. Phase Equilibria 1993, 14, 129-130. [CrossRef]

10. Herrmannsdörfer, T.; Fischer, P.; Mattenberger, K.; Vogt, O. Temperature dependences of rhombohedral lattice distortion and of ferromagnetic uranium ordering in the uranium monochalcogenides. J. Alloys Compd. 2006, 414, 14-19. [CrossRef]

11. Tougait, O.; André, G.; Bourée, F.; Noël, H. Neutron diffraction study of magnetic ordering of two binary uranium tellurides $\mathrm{U}_{3} \mathrm{Te}_{5}$ and $\mathrm{U}_{2} \mathrm{Te}_{3}$. J. Alloys Compd. 2001, 317-318, 227-232. [CrossRef]

12. Stöwe, K. Contributions to the Crystal Chemistry of Uranium Tellurides. III. Temperature-Dependent Structural Investigations on Uranium Ditelluride. J. Solid State Chem. 1996, 127, 202-210. [CrossRef]

13. Solvyanskikh, V.K.; Yarembash, E.I.; Ellert, G.V.; Eliseev, A.A. On the system U-Te. Izv. Akad. SSSR Ser. Neor. Materialy 1968, 4 , 543-545.

14. Solvyanskikh, V.K.; Rozanov, I.A.; Gracheva, N.V. The S-Te-U. Russ. J. Inorg. Chem. 1977, 22, 893-896.

15. Tougait, O.; Potel, M.; Noël, H. Characterization of the Binary Uranium and Thorium Tellurides $\mathrm{U}_{7} \mathrm{Te}_{12}$ and $\mathrm{Th}_{7} \mathrm{Te}_{12}$. Inorg. Chem. 1998, 37, 5088-5091. [CrossRef]

16. Tougait, O.; Potel, M.; Padiou, J.; Noël, H. Crystal structure and properties of the binary uranium telluride $\mathrm{U}_{2} \mathrm{Te}_{5}$. J. Alloys Compd. 1997, 262-263, 320-324. [CrossRef]

17. Boehme, D.R.; Nichols, M.C.; Snyder, R.L.; Matheis, D.P. An investigation of the tellutium-rich uranium tellurides using X-ray powder diffraction. J. Alloys Compd. 1992, 179, 37-59. [CrossRef]

18. Ellert, G.V.; Sevast'yanov, V.G.; Solvyanskikh, V.K. The Se-U and Te-U Systems. Russ. J. Inorg. Chem. 1975, 20, 120-124.

19. Czechowicz, D.G. Combustion Synthesis and Characterization of Uranium and Thorium Tellurides: LA-10559-T. Master's Thesis, Los Alamos National Laboratory, Los Alamos, NM, USA, 1985.

20. Wolf, A. Modellierungen zur Kristallzüchtung von CrSb2 und UPTe, Ein Beitrag zur Rationale Syntheseplanung; Springer: Wiesbaden, Germany, 2017. [CrossRef]

21. Westrum, E.F.; Gronvold, F. Chemical Thermodynamics of the Actinide Element Chalcogenides. In Proceedings of the Symposium on Thermodynamics of Nuclear Materials (IAEA), Vienna, Austria, 21-25 May 1962.

22. Czechowicz, D.G. A Study of vaporization Thermodynamics in the Uranium-Tellurium System: LA-10621-T. Master's Thesis, Los Alamos National Laboratory, Los Alamos, NM, USA, 1986.

23. Baskin, Y.; Smith, S.D. Enthalpy of formation data on compounds of uranium with groups VA and VIA elements. J. Nucl. Mater. 1970, 37, 209-222. [CrossRef]

24. Mills, K.C. Thermodynamic Data for Inorganic Sulphides, Selenides and Tellurides; Butterworth \& Co.: London, UK, 1974.

25. Qian, S.; Qiu, R.; Tang, J.; Chen, J.; Liu, P.; Ao, B. Theoretical Assignment of Oxidation State of Uranium in Binary, Ternary, and Quaternary Tellurides. J. Phys. Chem. 2021, 125, 1029-1040. [CrossRef]

26. Saal, J.E.; Kirklin, S.; Aykol, M.; Meredig, B.; Wolverton, C. Materials Design and Discovery with High-Throughput Density Functional Theory: The Open Quantum Materials Database (OQMD). JOM 2013, 65, 1501-1509. [CrossRef]

27. Solvyanskikh, V.K.; Ellert, G.V.; Yarembash, E.S. Transport mechanism and kinetics in Uranium chalcogenides. Izv. Akad. SSSR Ser. Neor. Mater. 1967, 3, 1133.

28. Sevast'yanov, V.G.; Solvyanskikh, V.K.; Ellert, G.V. Equilibria in the $\mathrm{US}_{x}-\mathrm{Br}_{2}$ and $\mathrm{USe}_{x}-\mathrm{Br}_{2}$ system. Zh. Neorg. Khim. 1971, 16, 3357.

29. Dinsdale, A.T. SGTE data for pure elements Calphad 1991, 15, 317-425. [CrossRef]

30. Hillert, M.; Jansson, B.; Sundman, B.; Agren, J. A two-sublattice model for molten solutions with different tendency for ionization Metall. Trans. 1985, 16, 661. [CrossRef]

31. SSUB5: SGTE Substances Database, Version 5.2. Available online: https://thermocalc.com/products/databases/general-alloysand-pure-substances/ (accessed on 15 January 2016).

32. Guillaumont, R. Update on the Chemical Thermodynamics of Uranium, Neptunium, Plutonium, Americium and Technetium; OECD Nuclear Energy Agency, Data Bank: Issy-les-Moulineaux, France, 2003.

33. Chatterjee, N.D.; Krüger, R.; Haller, G.; Olbricht, W. The bayesian approach to an internally consistent thermodynamic database : theory, database, and generation of phasediagrams. Contrib. Mineral. Petrol. 1998, 133, 149-168. [CrossRef]

34. Duong, T.C.; Hackenberg, R.E.; Landa, A.; Honarmandi, P.; Talapatra, A.; Volz, H.M.; Llobet, A.; Smith, A.I.; King, G.; Bajaj, S.; et al. Revisiting thermodynamics and kinetic diffusivities of uranium-niobium with bayesian uncertainty analysis. Calphad 2016, 55, 219-230. [CrossRef]

35. Lawrence, E. Reconstruction Fonctionnelle et Analyse D'incertitude dans le Cadre d'un Problème Inverse de Thermodynamique Chimique. Ph.D. Thesis. Université Paul Sabatier Toulouse 3, Toulouse, France, 2020.

36. Robert, C.P. The Bayesian Choice: A Decision-Theoretic Motivation; Springer: New York, NY, USA 1994.

37. Azaïs, J.M.; Bardet, J.M. Le Modèle Linéaire par L'exemple. Régression, Analyse de la Variance et Plans d'Expériences. Illustrations Numériques avec les Logiciels R, SAS et Splus; Dunod: Paris, France, 2006.

38. Sundman, B.; Lu, X.G.; Ohtani, H. The implementation of an algorithm to calculate thermodynamic equilibria for multi-component systems with non-ideal phases in a free software. Comput. Mater. Sci. 2015, 101, 127-137. [CrossRef]

39. The OpenCalphad Repository. Available online: http://github.com/sundmanbo/opencalphad (accessed on 12 December 2018). 